The Israeli Journal of Aquaculture IJA.73.2021.1531108, 18 pages

CCBY-NC-ND-4.0 • https://doi .org/10.46989/001c.29485

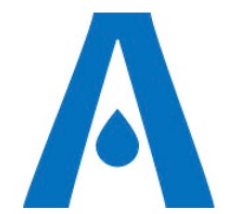

The $I J A$ is a peer-reviewed open-access, electronic journal, freely available without charge to users

Produced by the AquacultureHub non-profit Foundation Sale of $I J A$ papers is strictly forbidden

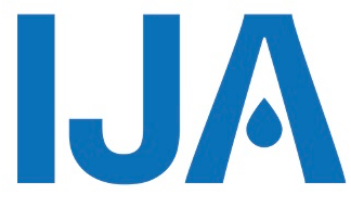

\title{
Characterization and expression analysis of heat shock protein $90 \beta$ from golden pompano (Trachinotus ovatus, Linnaeus 1758 ) in responses to stock density stress and Cryptocaryon irritans infection
}

\author{
Hua-Yang Guo ${ }^{1,2}$, Quan Yang 1,2, Bo Liu,1,2, Bao-Suo Liu' ${ }^{1,2}$, Nan Zhang ${ }^{1,2}$, Liang \\ Guo ${ }^{1,2}$, Ke-Cheng Zhu ${ }^{1,2}$, Dianchang Zhang ${ }^{1,2,3,4 *}$
}

${ }^{1}$ Key Laboratory of South China Sea Fishery Resources Exploitation and Utilization, Ministry of Agriculture and Rural Affairs; South China Sea Fisheries Research Institute, Chinese Academy of Fishery Sciences, 510300, Guangzhou, Guangdong Province, China

2 Tropical Aquaculture Research and Development Center, South China Sea Fisheries Research Institute, Chinese Academy of Fishery Sciences, 572018, Sanya, Hainan Province, China

${ }^{3}$ Guangdong Provincial Engineer Technology Research Center of Marine Biological Seed Industry, Guangzhou, Guangdong Province, China

${ }^{4}$ Guangdong Provincial Key Laboratory of Fishery Ecology and Environment, Guangzhou, Guangdong Province, China

Keywords: Trachinotus ovatus, HSP90ß, Stocking density, Cryptocaryon irritans

\begin{abstract}
Heat shock protein 90 family, a class highly conserved protein and important molecular chaperone and its plays a role in enhancing animal adaptability to environmental stress and improving immunity. In this study, a full-length HSP90 $\beta$ cDNA (ToHSP90 $\beta$ ) sequence was identified from Trachinotus ovatus. The cDNA of ToHSP90 $\beta$ was 2648 base-pairs (bp) long and contained a $5^{\prime}$-terminal untranslated region of $130 \mathrm{bp}$, a $3^{\prime}$-terminal untranslated region of $334 \mathrm{bp}$, and the open reading frame length $2184 \mathrm{bp}$, which can encode 728 amino acids. The ToHSP90 $\beta$ has a heat shock protein 90 family signature. The ToHSP90 $\beta$ amino acid sequence has high homology with other fish HSP9O $\beta$ amino acids (92.5-99.17\%) and contains five conserved signal regions and a structural feature of the cytoplasmic HSP90 family. Phylogenetic tree analysis indicated that ToHSP90 $\beta$ was most closely related to Seriola dumerili HSP90 $\beta$. Tissues expression analysis showed that the ToHSP90 $\beta$ was expressed in all nine tissues of $T$. ovatus, and its expression was the highest in the intestine. The stocking density significantly affected the expression of ToHSP9O $\beta$ in the kidney and brain tissues, and its expression levels increased significantly with increasing stocking densities, indicating that it plays an important regulatory role in the process of $T$. ovatus' chronic stress response. After Cryptocaryon irritans infection, the mRNA expression of ToHSP90 $\beta$ was significantly increased in liver and kidney tissues, indicating that ToHSP9O $\beta$ would be involved in the immune regulatory response. This study was the first to analyze the expression pattern of HSP90 $\beta$ mRNA in $T$. ovatus tissues and their expression responses to stocking density stress and $C$. irritans infection. To provide a theoretical basis for further study of the function of fish HSP90 and its physiological mechanism in environmental stress and parasite infection.
\end{abstract}

\section{Introduction}

* Corresponding author. Dr. Dian C. Zhang. E-mail address: zhangdch@scsfri.ac.cn 
Heat shock proteins (HSPs), also known as stress proteins, are a class highly conserved family of cellular proteins and important molecular chaperones that are widely found in living organisms and divided into two types of constitutive and inductive (Gao et al., 2008; Roberts et al., 2010; Yebra-Pimentel et al., 2019; Zahedi et al., 2019). Heat shock proteins play an important role in protecting fish from stress injuries (Jun et al., 2015). Synergistic effects of genetic characteristics and external factors affect the regulation of heat shock proteins in fish (Iwama et al., 1999). Under adverse stress conditions, the body will synthesize heat shock proteins through physiological and biochemical reactions to protect itself from damage to the stressors (Pierce et al., 2013; Lin et al., 2018). Yebra-Pimentel et al. (2019) showed that proteins are misfolded and accumulated in the cytoplasm due to unfavorable stress, resulting in a highly conserved heat shock response to maintain protein stability. Lin et al. (2018) stated that HSPs protect proteins and cells from damage under unfavorable stress conditions. In addition, HSPs also play an important role in immune response, apoptosis, and inflammation (Roberts et al., 2010). Some scholars believe that HSPs are indicators of stress and are important biomarkers for a variety of abiotic variables (Sanders, 1993; Song et al., 2018; Vargas-Chacoff et al., 2019).

According to molecular size and sequence homology, heat shock proteins can be divided into five families: HSP110, HSP90, HSP70, HSP60 and low molecular weight Heat shock proteins (Csermely et al., 1998). Heat shock protein 90 family (HSP90), one of the important members of the heat shock proteins family, accounting for about $1 \%-2 \%$ of total cell soluble protein under non-stress conditions (Csermely et al., 1998; Sreedhar et al., 2004). In recent years, HSP90 has received considerable attention in aquatic animal research. As a stress protein and molecular chaperone, HSP90 plays an important biological role in biological tolerance to various adversity stressors (Young et al., 2001; Fu et al., 2011). HSP90 expression levels are up-regulated by a series of stressors such as changes in salinity, temperatures, heavy metals and ammonia nitrogen as well as bacterial infections (Deane et al., 2002; Qin et al., 2016; Shin et al., 2018). In the stress response of cells, HSP90 interacts with proteins that change their conformation due to environmental stimuli, ensuring proper folding of proteins and preventing non-specific aggregation of proteins, and enhancing the ability of animals to adapt to environmental stimuli (Zhang et al., 2009; Lin et al., 2018). Simultaneously, HSP90 is involved in the regulation of apoptosis or necrosis as well as signal transduction of various hormones and growth factor receptors (Pratt, 1998; Sreedhar et al., 2004). In addition, HSP90 also maintains key proteins by forming a specific complex, such as protein kinases and steroid receptors (Csermely et al., 1998). The HSP90 family can be divided into five subfamilies: the mitochondrial HSP90 homolog tumor necrosis factor regulatory protein 1(TRAP1), the chloroplast HSP90 subtype, the cytosolic HSP90a (HSP90AA) and HSP90ß (HSP90AB), the endoplasmic reticulum HSP90B subtype glucose-regulated protein 94 (Grp94) and hightemperature protein G (HtpG) in bacteria (Song et al., 1995; Patel et al., 2015; Du et al., 2019). At present, the most extensive studies on HSP90a and HSP90 $\beta$ in the cytoplasm. Some scholars believe that HSP90 $\beta$ is a subtype of the HSP9O family that has undergone a long time of cell evolution and adaptation, while HSP90a is a rapidly responding subtype of cell protection, and HSP90 $\beta$ is more suitable for the study of chronic stress than HSP90a (Kim et al., 2013). To date, the HSP90 genes were isolated and identified in many teleosts such as Solea senegalensis (Manchado et al., 2008), Epinephelus coioides (Chen et al., 2010), Ctenopharyngodon idella (Wu et al., 2012), Miichthys miiuy (Wei et al., 2013), Botia reevesae (Qin et al., 2016), Channa argus (Zhou et al., 2017) and Megalobrama amblycephala (Song et al., 2018).

The success farming of commercial fish depends on the provision of appropriate environmental factors to maintain the health of the fish (Kim et al., 2013). The health and welfare of farmed fish is of widespread concern in society, such as the stocking density. Stocking density is considered to be an important factor in determining fish production and economic benefits in fish farmed practices (Kim et al., 2013; Refaey et al., 2018). It is well known that high stocking densities can lead to chronic stress and reduce fish welfare in intensive aquaculture (Vazzana et al., 2002). Zahedi et al. (2019) showed that high stocking density as a chronic stress source had multiple effects on the physiology and 
welfare of farmed fish, in primary, secondary and tertiary stress responses. The mRNA synthesis of encoding "stress-related proteins" is a primary response, and its expression can be measured by real-time fluorescence quantitative PCR (Gornati et al., 2004). Terova et al. (2005) pointed out that molecular biomarkers that indicate gene activity can serve as important early indicators of response to stress in organism. As a specific anti-stress protein, HSP90 plays an important role in enhancing animal adaptation to environmental stressors (Lin et al., 2018). Studies have shown that the expression level of HSP90 mRNA was significantly changed in some tissues of fish under high stocking density stress, such as Dicentrarchus labrax (Gornati et al., 2004), Takifugu obscurus (Kim et al., 2013), Acipenser schrenckii (Ni et al., 2014) and Paralichthys adspersus (Valenzuela et al., 2019). In this study, we investigated the expression responses of HSP90 $\beta$ gene in golden pompano under high stocking density stress.

The non-specific response caused by the adverse stimulation of external environmental factors is called a stress response. Stress responses are applicable to a wide range of physiological mechanisms. In teleosts, the kidney and liver are important immune tissues (Press and Evensen, 1999). Yang et al. (2020) showed that the expression of HSP90a in the liver and kidney tissues of golden pompano was increased after Cryptocaryon irritans infection. Wang et al. (2017) showed that the HSP90 mRNA level of Hyriopsis cumingii was significantly regulated by changes in temperature, cadmium and Aeromonas hydrophila. Therefore, HSP90 is involved in regulating the cellular stress response and plays a key role in protecting organisms from external environmental stress.

The golden pompano (Trachinotus ovatus, Linnaeus 1758), belongs to Trachinotus and Carangidae, and is widely distributed in tropical and subtropical waters of Southeast Asia and the Mediterranean. Due to its rapid growth, delicious meat quality and high nutritional value, and has become a most important cultured marine fish in the southeast coast of China (Tutman et al., 2004; Liang et al., 2018). However, due to the increasing stocking density and parasites, affecting their growth, and even causing economic losses. This study was to analyze the cDNA structure and characterization of the HSP90 $\beta$, and to investigate the expression and distribution of the HSP90 $\beta$ mRNA in various tissues of $T$. ovatus and its expression in three different stocking densities and $C$. irritans, to further study the molecular mechanism of HSP90 $\beta$ gene under environmental stress to provide basic data.

\section{Materials and Methods}

Experimental materials and design. The experimental golden pompano was obtained from the Tropical Fisheries Research and Development Centre, South China Sea Fisheries Research Institute, Chinese Academy of Fishery Sciences, Xincun Town, Lingshui, China. The experimental fish were kept for one week before the experiment to adapt to the rearing environment in the sea cages of Xincun Town. After one week, the experimental fish (weight: $9.75 \pm 0.11 \mathrm{~g}$ ) were randomly assigned to the corresponding cages (specification: $1 \mathrm{~m} \times 1 \mathrm{~m} \times 1 \mathrm{~m}$ ) according to 100, 200 and 300 fish, marked as low stocking density (LD), medium stocking density (MD) and high stocking density (HD), three replicates each experimental group. The experiment lasted for 10 weeks, observed, and record the survival status of the fish every day. The design and results of the stocking density experiment were previously described by Yang et al (2020).

At the end of the experiment, fish were fasted for one day and were anaesthetized with 100 mg / L eugenol (Shanghai Medical Devices Co., Ltd., Shanghai, China). The liver, kidney, brain, and muscle of three fish were collected in each cage and immediately placed in liquid nitrogen for freezing. The samples of the liver, spleen, intestine, kidney, gill, brain, fins, blood, and muscle tissues from three healthy golden pompano (non-experimental fish) were collected and frozen in liquid nitrogen and stored at $-80^{\circ} \mathrm{C}$ until total RNA was isolated.

Healthy $T$. ovatus with an average body weight of $98 \pm 15 \mathrm{~g}$ were purchased from Lingshui Marine Fish Farm in Hainan Province, China, where no outbreaks of infection had emerged during the breeding period. 200 fishes were randomly selected and examined; no parasites were identified on their gills, fins, or skin. The selected fishes were acclimated 
in two fiberglass aquariums with $1000 \mathrm{~L}$ water volume for two weeks and fed twice times a day (8:00 and 17:00) with commercial feed prior to infection. The experiment was maintained in fresh seawater at $28 \pm 2{ }^{\circ} \mathrm{C}$ with 30\%o salinity. 100 healthy fish were infected with C. irritans at a dose of 600 theronts / fish in the experimental group (EG), and 100 healthy fish were used as a control group (CG), and no infection treatment was performed under the same reared conditions. The experiment lasted three days. The $C$. irritans used in this study were isolated from the naturally diseased $T$. ovatus and subcultured in the laboratory. After $C$. irritans challenge for $0,6,12,24,48$ and $72 \mathrm{~h}$, the liver, kidney, and gill of six fish were collected in the EG. The same tissues were collected from six healthy fish as a negative control in the CG. Fish were anaesthetized before collecting samples. All samples (liver, kidney, and gill) were collected in $2 \mathrm{~mL}$ cryotubes and rapidly frozen in liquid nitrogen.

All the experiments in this study were approved by the Animal Care and Use Committee of South China Sea fisheries Research Institute, Chinese Academy of fishery Sciences (no.SCSFRI96-253) and performed according to the regulations and guidelines established by this committee.

Total RNA extraction and CDNA synthesis. Total RNA was isolated from the liver, kidney, brain, muscle, gill, and fin tissues of the experimental fish and nine tissues of the healthy fish according to the instructions of HiPure Universal RNA Mini kit (Magen, Guangzhou, China). The RNA quality and concentration were measured by $1.0 \%$ agarose gel electrophoresis and NanoDrop 2000 (Thermo Scientific, USA), respectively. The better isolated RNA was reverse transcribed into cDNA for qPCR using the PrimeScript ${ }^{T M}$ RT reagent kit with gDNA Eraser (TaKaRa, Dalian, China). The synthesized cDNA samples were stored at $-80{ }^{\circ} \mathrm{C}$ until use.

Cloning and sequencing. T. ovatus HSP90 3 genomic sequences was obtained by whole genome sequencing, assembled, and annotated (Accession No. PRJEB22654 under ENA; Sequence Read Archive under BioProject PRJNA406847) in our laboratory. The cDNA sequence was obtained by random sequencing technique and found to be highly homologous with HSP90 $\beta$ mRNA, complete CDS in other fish by comparison in GenBank database, named ToHSP90 $\beta$. Primers ToHSP90ß-F/R were designed by Primer Premier 5.0 software to validated open reading frame (ORF). The target fragment was amplified by using cDNA as templates, and the target fragment was purified and ligated with a vector (pMDTM18-T vector cloning kit, TaKaRa) to transform competent cells. Cloning and screening of white colonies, then PCR reaction and sequencing (Beijing Ruibo Xingke Biotechnology Co., Ltd., Beijing, China.).

Bioinformatic analysis. The sequencing results were aligned in BLAST of the Genbank database (https://blast.ncbi.nlm.nih.gov/Blast.cgi). The open reading frame is predicted by ORFfinder (https://www.ncbi.nlm.nih.gov/orffinder/) and speculated on the amino acid sequence. The molecular weight (Mw) and isoelectric point ( $P I)$ values were calculated by the ExPASy program (https://web.expasy.org/compute_pi/). Signal peptides, glycosylation sites, and protein phosphorylation sites on the amino acid sequence were predicted using the ExPASy (http://prosite.expasy.org/), NetNGly (http://www.cbs.dtu.dk/services/NetNGlyc/) and Kinasephos program (http://kinasephos.mbc.nctu.edu.tw/), respectively. The signal peptides were predicted to useing SignalP 4.1 (http://www.cbs.dtu.dk/services/). The conserved domain was speculated by SMART software (http://smart.emblheidelberg.de/). Protein secondary structure prediction using the GOR method (https://npsa-prabi.ibcp.fr/cgibin/npsa_automat.pl?page=/NPSA/npsa_phd.htmI). Meanwhile, the three-level structure prediction was performed using the SWISS-MODEL software (http://swissmodel.expasy.org/). The HSP9O $\beta$ sequences of other species were obtained from the NCBI database. Multiple sequence alignments by using the Clustal Omega program (https://www.ebi.ac.uk/Tools/msa/clustalo/), and phylogenetic trees were constructed using MEGA7.0 software.

Quantitative real-time PCR. Based on the CDNA sequence of ToHSP90 $\beta$, the upstream and downstream specific primers (ToHSP90 $3-q F / q R$ ) of ToHSP90 $\beta$ were designed using the Primer Premier 5.0 software and the reference gene elongation factor 1 alpha (EF-1a F/R) 
(Table 1). The qPCR reaction was performed on the Roche LightCycler ${ }^{\circledR} 480$ II (Roche Diagnostics, Shanghai, China) according to the TB Green ${ }^{T M}$ Premix Ex Taq ${ }^{T M}$ II (TaKaRa) instructions. A $12.5 \mu \mathrm{L}$ reaction volume contained $6.25 \mu \mathrm{L} 2 \times$ TB Green Premix Ex Taq II (Tli RNaseH Plus) (TaKaRa), $1 \mu \mathrm{L}$ cDNA template, $0.5 \mu \mathrm{L}$ each primer of forward and reverse and $4.25 \mu \mathrm{L}$ of Milli-Q water. The thermal profile for qPCR was $94^{\circ} \mathrm{C}$ for $30 \mathrm{~s}$, followed by 40 cycles at $94^{\circ} \mathrm{C}$ for $5 \mathrm{~s}, 60^{\circ} \mathrm{C}$ for $20 \mathrm{~s}$. Assay of all samples was repeated three times. The relative expression of ToHSP90 $\beta$ in the liver, kidney, brain and muscle tissues of the experimental fish and nine tissues of the healthy fish were calculated by the $2^{-\Delta \Delta c t}$ method (Livak and Schmittgen 2001). All data were analysed according to one-way analysis of variance (ANOVA) and Tukey's post hoc test in this study using SPSS 22.0 software (IBM, New York, USA). Results are expressed as mean \pm SE (mean \pm standard error). Significant difference was considered at $P<0.05$.

Table 1 Primers used in this study.

\begin{tabular}{|c|c|c|}
\hline Primer name & primer sequences $\left(5^{\prime}-3^{\prime}\right)$ & application \\
\hline ToHSP90ß-F & САTTTCCCTGCGTCAGAGAG & \multirow{2}{*}{$\begin{array}{l}\text { verify open } \\
\text { reading frame }\end{array}$} \\
\hline ToHSP90ß-R & TCAGGGGGGTGGGTAGATTT & \\
\hline ToHSP90ß-qF & GTTTCTACTCTGCCTACCTTGTG & \multirow{2}{*}{ qRT-PCR } \\
\hline ToHSP90ß-qR & TСTССTTGАСССТСТTСТСС & \\
\hline$E F-1 a F$ & CCCCTTGGTCGTTITGCC & \multirow{2}{*}{ Reference gene } \\
\hline$E F-1 a R$ & GCCTTGGTTGTCTTTCCGCTA & \\
\hline
\end{tabular}

\section{Results}

The CDNA sequence characteristics of ToHSP9OB. As shown in Figure 1, the full-length ToHSP90 $\beta$ cDNA was 2648 base-pairs (bp) (GenBank accession number: MN641479), including a complete open reading frame with the start codon ATG located 131-133 and the stop codon TAA located 2315-2317. 5'-terminal untranslated region (5'-UTR) of 130 $\mathrm{bp}$, open reading frame (ORF) length $2184 \mathrm{bp}$, and a $3^{\prime}$-terminal untranslated region (3'UTR) of $334 \mathrm{bp}$. The ORF finder program predicts that the ORF encodes a protein of 728 amino acids. Its theoretical molecular weight of $83.681 \mathrm{kDa}$, theoretical isoelectric point of 4.90, and no signal peptide sequence was detected at the $\mathrm{N}$-terminal. Fourteen phosphorylation sites ( 7 serine sites, 1 threonine site, and 6 tyrosine sites, respectively) were predicted by the kinasephos program, and no glycosylation sites were detected (Figure 1). The Heat shock protein 90 family signature (YSNKEIFLRE) sequence was predicted at $32-41$ by ExPASy program and using the SMART program to predict that ToHSP90 $\beta$ has a histidine kinase-like ATPases domain at 34-188 (Figure 1). According to the multiple alignment of the amino acid sequences of $T$. ovatus and other fishes, it was found that there were five conserved signal regions of the HSP90 family, and the cytosolic HSP90 family signature MEEVD (Figure 2). The above analysis indicates that it is indeed a member of the HSP9O family.

The prediction of the secondary structure of ToHSP90 $\beta$ amino acid sequences by GOR method, showed that its secondary structure consisted of $46.57 \%$ alpha helix, $13.60 \%$ extended strand and $39.84 \%$ random coil. A three-dimensional construction model of the ToHSP90 $\beta$ protein was constructed by the SWISS-MODEL program (Figure $\mathbf{3}$ ). The results showed that the ToHSP9O $\beta$ protein consisted of 21 a-helices and $22 \beta$-sheets and connected random coils.

Homology analysis of ToHSP9Oß. Multiple alignment analysis of amino acid sequences using the Clustal Omega program (Table 2), showed that the amino acid sequence of the ToHSP90 $\beta$ has high homology with other osteichthyes (92.51-99.17\%), which has the highest homology with S. dumerili $(99.17 \%)$, followed by E. naucrates $(98.90 \%)$, and it has the lowest homology with S. salar (92.51\%). The homology of amino acid sequence of the ToHSP90 $\beta$ with mammals, aves, amphibia and reptiles are 90.97-91.25\%, 90.56$91.11 \%, 91.52 \%$ and $89.47 \%$, respectively. The Poisson Correction distances of the ToHSP90 $\beta$ amino acids and other osteichthyes, mammals, aves, amphibia and reptiles are 
$0.008-0.074,0.086-0.089,0.086-0.092,0.081,0.103$, respectively. The closest pairwise distance between $T$. ovatus and $S$. dumerili is 0.008 , and the furthest pairwise distance with $O$. hannah is 0.103 .

CTGAAACGCATGTGTATAAAGATTCCGGTCTATTAAAAGGAAGTCCCTTTCCATTTCCCTGCGTCAGAGAGCAGAAGGGACAGCACATTG GTTGCATTTTATTCAAGAAAAGTCAACACAAATAAACAAGatgcctgaagaaatgcaccaagaggaggaggctgagacct t tgecttcca $\begin{array}{lllllllllllllllllllllll}M & P & E & E & M & H & Q & E & E & E & A & E & T & F & A & F & Q\end{array}$ ggcagagattgctcagctgatgtccetgatcatcaacaccttctactccaacaaagagatcttcctcagggagttgatctctaatgcttc

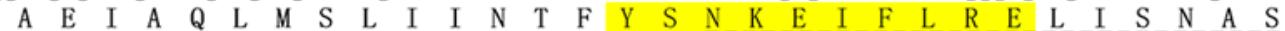
tgatgctttggacaaaatccgctatgaaagcctgactgacccttccaagctggacagcggcaaggatctgaaaattgacatcatccccaa D caaagctgaccgtaccctgaccctcatcgacactggaatcggcatgaccaaagctgacctgat taacaacctgggtaccatcgccaagtc

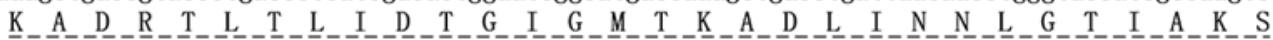
tggcactaaggccttcatggaggecctgcaggctggagctgacatctccatgattggtcagt tygtgtgggtt tctactctgcctacct $\underline{G} \_$I_K $\_$A $\_$_ $\_M$ tgtggctgagagggtggtt tcatcaccaaacacaacgatgatgagcagtatgcctgggagtcctctgctggcggttcct tcaccgtcaa

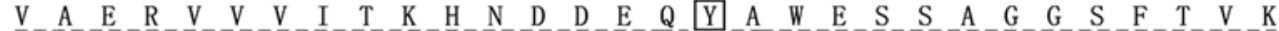
ggttgacaacggtgagcccattggccgtggaacaaagatcgtcttgtacctgaaggaggaccagacagagtacattgaggagaagagggt

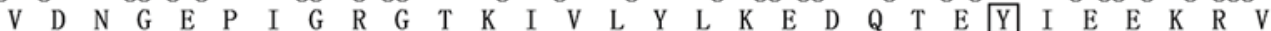
caaggagattgtaaagaagcactctcagttcatcggctaccccatcaccctgtt tgtggagaaggagcgcgacaaggagatcagtgacga

$\begin{array}{llllllllllllllllllllllllllllll}K & E & I & V & K & K & H & S & Q & F & I & G & Y & P & I & T & L & F & V & E & K & E & R & D & K & E & I & S & D & D\end{array}$ cgaggcagaggaggaaaaggcagagaaggaagagaaggaagagaaagaggacggtgaggacaagccaaagat tgaggatgtgggctcaga

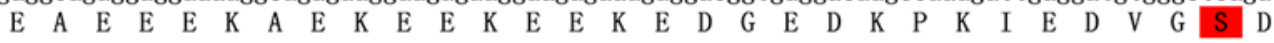
tgatgaggaggactccaaagacaaggacaagaagaagaaaaagaagatcaaggagaagtacattgaccaggaggagctgaacaagaccaa

$\begin{array}{lllllllllllllllllllllllllllllll}D & E & E & D & S & K & D & K & D & K & K & K & K & K & K & I & K & E & K & Y & I & D & Q & E & E & L & N & K & T & K\end{array}$ gcccatctggaccagaaaccctgatgacatcacaaacgaggaatatggcgagttctacaagagtctgaccaatgactgggaggatcacct

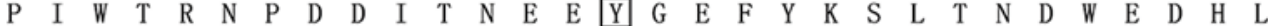
cgctgtcaagcatttctcagtggagggccagcttgagttccgcgccetgctcttcattccccgtcgtgcaccttttgacctctttgagaa

$\begin{array}{llllllllllllllllllllllllllllll}\text { A } & V & K & H & F & S & V & E & G & Q & L & E & F & R & A & L & L & F & I & P & R & R & A & P & F & D & L & F & E & N\end{array}$ caagaaaaagaagaataacatcaagctgtacgtgaggagagtctttatcatggacaactgtgaagaactcatcccagagtacctgaactt

$\begin{array}{llllllllllllllllllllllllllllll}K & K & K & K & N & N & I & K & L & Y & V & R & R & V & F & I & M & D & N & C & E & E & L & I & P & E & Y & L & N & F\end{array}$ tgtccgtggtgtggtggactcagaggacctgcccctcaacatctcaagagaaatgctgcagcagagcaagatcctcaaggtcattcgcaa

$\begin{array}{llllllllllllllllllllllllllllll}V & R & G & V & V & D & S & E & D & L & P & L & N & I & S & R & E & M & L & Q & Q & S & K & I & L & K & V & I & R & K\end{array}$ gaacattgtcaagaagtgtctggagctctttgctgaactggctgaggataaggagaattacaagaagttctatgaaggattctccaagaa $\begin{array}{llllllllllllllllllllllllllllll}N & I & V & K & K & C & L & E & L & F & A & E & L & A & E & D & K & E & N & Y & K & K & F & Y & E & G & F & S & K & N\end{array}$ catcaagctgggtatccatgaggactctcaaaaccgcaagaagttgtccgagctgctgcgttaccacagctcccagtctggagatgagat

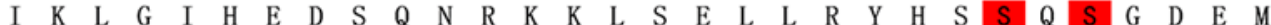
gacctccctcacagagtacctttcc gcatgaaggaaaaccagaagtccatctactacat tactggtgagagcaaggatcaggtggccaa

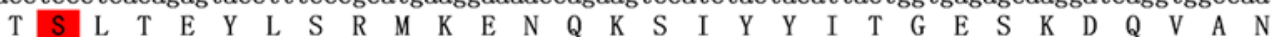
ctccgcttttgtcgagcgtgtccgcaagcgtggctttgaggtcctgtacatgacagagcccattgatgagtactgtgtccagcagttgaa $\begin{array}{lllllllllllllllllllllllllllllll}S & A & F & V & E & R & V & R & K & R & G & F & E & V & L & Y & M & T & E & P & I & D & E & Y & C & V & Q & Q & L & K\end{array}$ ggagtttgatggcaagagcctggtctctgtcaccaaagagggcctggagctgccagaggatgaggaggagaagaaaaagatggaggagga

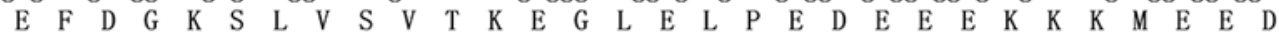
caaggccaagttcgagagcctctgcaagctcatgaaggagatccttgacaagaaagtggagaaggtgacagtgtccaacagactggtgtc

$\begin{array}{llllllllllllllllllllllllllllll}K & A & K & F & E & \text { S } & \text { L } & C & K & \text { L } & M & K & \text { E } & \text { I } & \text { L } & \text { D } & K & K & \text { V } & \text { E } & K & \text { V } & \text { T } & \text { V } & \text { S } & \text { N } & \text { R } & \text { L } & \text { V } & \text { S }\end{array}$ ttcaccctgctgcattgtgacaagtacttacggctggacggccaacatggagaggatcatgaaggcccaggcactcagggacaactccac

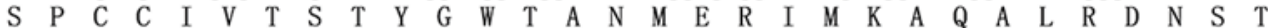
catgggetacatgatggccaagaagcacctggagatcaaccttgaccaccccatcgtggagactctcaggcagaaggecgacgctgacaa

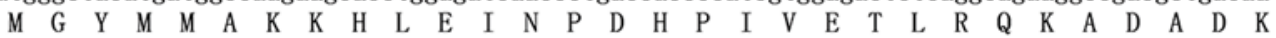
gaacgacaaggctgtgaaggacctcgtcatcctgctcttcgaaaccgccetgctgtcctcaggcttctccttggacgacccacagaccca

$\begin{array}{lllllllllllllllllllllllllllllll}N & D & K & A & V & K & D & L & V & I & L & L & F & E & T & A & L & L & S & S & G & F & S & L & D & D & P & Q & \text { (T) } & H\end{array}$ ctccaaccgcatctacagaatgatcaaacttgggctgggtatcgatgacgacgatgttcccacagaggaggccacttctacatccgtccc

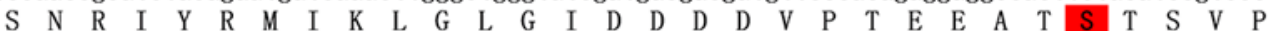
agatgagattcctcccctagaaggcgatggtgaagatgacgcttcccgtatggaagaagt tgattaaATCTACCCACCCCCCTGATTTCT $\begin{array}{lllllllllllllllllllll}D & E & I & P & P & L & E & G & D & G & E & D & D & A & S & R & M & E & E & V & D\end{array}$ AACACTTAGCCTCACTTTTCAATTGTTCATCCTTTAAAACTGCAGTAACTGCAAAACAAATAGTCATTCATGTTGTGTGGTGGACCAGTG TTGCTCTTGTGTCTAGAGCATTACTCTGCAAGACCTTTTAAGAAAAGCAGTTTTGGTTTTTGCTGTAATAAGTTCATGGTGACAGCACAT TTGTTTTAACAAGTACCCTGTTGCACTGAGTTTTAAATGTTGGAGTGGTAAATGTGTGAACATGGGAATGGTACATTCCATTATCAGGTC TGGAGGGTTTGGGAGGTTCTGCTCATGTGCAACACTGC

90 180 17 270 47 360 77 450 107 540 137 630 167 720 197 810 227 900 257 990 287 1080 317 1170 347 1260 377 1350 407 1440 437 1530 467 1620 497 1710 527 1800 557 1890 587 1980 617 2070 647 2160 677 2250 707 2340 728 2430 2520 2610 2648

Figure 1 Sequence of CDNA and predicted amino acid sequence of the ToHSP90 3 . The initiation codon (atg) is underlined. The stop codon (taa)is indicated by a thick line. The heat shock proteins 90 family signature sequence (YSNKEIFLRE) is indicated by a shade of yellow. The serine site is marked by a shade of red. The threonine site is represented by a circle. Tyrosine sites are indicated by boxes. The ToHSP9Oß histidine kinase-like ATPases domain is indicated by a dashed underline. The black and green numbers on the right side of the figure are indicate the number of bases and amino acids, respectively. 
S.salar

P.olivaceus

S.chuatsi

L.calcarifer

Tovatus

S.dumer

D.rerio

C.batrachus

S.salar

P.olivaceus

S.chuatsi

L.calcarifer

$\frac{\text { Tovatus }}{\text { S.dumerili }}$

D.rerio

C.batrachus

S.salar

P.olivaceus

S.chuatsi

L.calcarifer

Tovatus

S.dumerili

Drerio

C.batrachus

S.salar

P.olivaceus

S.chuatsi

L.calcarifer

Tovatus

S.dumerili

D.rerio

C.batrachus

S.salar

P.olivaceus

S.chuatsi

L.calcarifer

Tovatus

D.rerio

C.batrachus

MPEEMRQEEEAETFAFQAEIAQLMSLI INTFYSNKEIFLRELISNASDALDKIRYESLTDPTKLDNGKELKIDVIPNVEERTLTLIDTG I MPGEMHQEEEAETFAFQAEIAQLMSLI INTFYSNKEIFLRELISNASDALDKIRYESLTEPTKMDSGKDLKIDIIPNKEDRTLTLIDTG MPEEMHQEEEAETFAFQAEIAQLMSLI INTFYSNKEIFLRELISNASDALDKIRYESLTEPTKMDSGKDLKIDI IPNKADRTLTLIDTGI MPEEMHQEEEAETFAFQAEIAQLMSLIINTFYSNKEIFLRELISNASDALDKIRYKSLTDPSKLDSGKDLKIDI IPNKADRTLTLIDTGI MPEEMHQEEEAETFAFQAEIAQLMSLI INTFYSNKEIFLRELISNASDALDKIRYESLTDPSKLDSGKDLKIDIIPNKADRTLTLIDTGI MPEEMHQEEEAETFAFQAEIAQLMSLI INTFYSNKEIFLRELISNASDALDKIRYESLTDPSKLDSGKDLKIDI IPNKADRTLTLIDTGI MPEEMRQEEEAETFAFQAEIAQLMSLI INTFYSNKEIFLRELVSNASDALDKIRYESLTDPTKLDSGKDLKIDI IPNVQERTLTLIDTGI MPEEMRQEEEAETFAFQAEIAQLMSLI INTFYSNKE IFLRELISNASDALDKIRYESLTDPSKLDSGKDLKIDIIPNKHERTLTI IDTGI

GMTKADLINNLGTIAKSGTKAFMEALQAGADISMIGQFGVGFYSAYLVAERVTVITKHNDDEQY I WESSAGGSFTVKVDTGEPMLRGTKV GUTKADLINNLGTIAKSGTKAFMEALQAGADISUIGQFGVGFYSAYLVAEKVVVITKHNDDEQYAWESSAGGSFTVKVDTGEPIGRGTKI GMTKADLINNLGTIAKSGTKAFMEALQAGADISMIGQFGVGFYSAYLVAERVVVITKHNDDEQYAWESSAGGSFTVKVDNGEPIGRGTKI GMTKADLINNLGT IAESGTKAFMEALQAGADISMIGQFGVGFYSAYLVAEKVVVITKHNDDEQYAWESSAGGSFTVKVDNGEPVGRGTKI GMTKADLINNLGTIAKSGTKAFMEALQAGADISMIGQFGVGFYSAYLVAERVVVITKHNDDEQYAWESSAGGSFTVKVDNGEPIGRGTKI GMTKADLINNLGTIAKSGTKAFMEALQAGADISMIGQFGVGFYSAYLVAEKVVVITKHNDDEQYAWESSAGGSFTVKVDSGEPIGRGTKI GMTKADLINNLGT IAKSGTKAFMEALQAGADISMIGQFGVGFYSAYLVAEKVTVITKHNDDEQYAWESSAGGSFTVKVDHGEPIGRGTKV GMTKADLINNLGTIAKSGTKAFMEALQAGADISMIGQFGVGFYSAYLVAEKVVVITKNNDDEQYAWESSAGGSFTVRVDHGEPIGRGTKV

ILHMKEDQTEYVEEKRVKEVVKKHSQFIGYPITLFVEKEREKEISDDEEEKAEEEK----EEKEAEDKPKIEDVGSDDEED-SKDKDKKK 265 VLHLKEDRTEYVEDKRVKEIVKKHSQFIGYPITLFVEKECDKEISDDEAEEEKAEK---EEKEDGEDKPKIEDVGSDDEED-SKDKDKKK 266 ILHLKEDQTEYIEEKRVKEIVKKHSQFIGYPITLFVEKERDKEISDDEAEEEKAEK---EEKEEGEDKPKIEDVGSDDEED-SKDKDKKK 266 ILYLKEDQTEYIEEKRIKEIVKKHSQFIGYPITLFVEKERDKEISDDEAEEEKAEK---EEKEDGEDKPKIKDVGSDDEED-SKDKDKKK 266 VLYLKEDQTEY IEEKRVKEIVKKHSQFIGYPITLFVEKERDKEISDDEAEEEKAEKEEKEEKEDGEDKPKIEDVGSDDEED-SKDKDKKK 269 ULYLKEDQTEY IEEKRVKEIVKKHSQFIGYPITLFVEKERDKEISDDEAFEEKAEKE--EKEDAFDKPKIEDVGSDDEED-SKDKDKKK 266 ILYLKDQTEYEKR IEKRVEVVKKHSQFIGYPITLYVEKERDKEISDDEAEEEKAEKEE--KEEEGEDKPKIEDVGSDDEED-TKDKDKKK 267 ILHLKEDQTEYIEEKRVKEVVKKHSQFIGYPITLYVEKERDKEISDDEAEEEKAEKEE--KEEEGEDKPKIEDVGSDDEED-TKDKDKKK 267
VLHLKEDQTEYIEEKRVKEVVKKHSQFIGYPITLFVEKERDKEISDDEAEDEKEEKEEKEEEEEGEDKPKIEDVGSDDEEDSSKDKDKKK
270

TKKIKEKYIDQEELNKTKPIWTRNPDDITMEEYGEFYKSLTNDWEEHLAVKHFSVEGQLEFRALLFIPRRAPFDLFENKKKKNNIKLYVR 355 KKKIKEKYIDQEELNKTKPIWTRNPDDITNEEYGEFYKSLTNDWEDHLAVKHFSVEGQLEFRALLFIPRRAPFDLFENKKKKNNIKLYVR 356 TKKIKEKYIDQEEL NKTKP IWTRNPDDITNEEYGEFYKSLTNDWFDHLAVKHFSVFGQLFRALLFIPRRAPFDLFENKKKKNNIKLYVR 356 KKKIKEKYIDOEELNTKPIWTRNPDDITNEEYGEFYKSLTNDWEDHUAVKHFSVEGOLEFRALLFIPRRAPFDLFENKKKKNNIKLYVR 356 KKKIKEKYIDQEELNKTKPIWTRNPDDITNEEYGEFYKSLTNDWEDHLAVKHFSVEGQLEFRALLFIPRRAPFDLFENKKKKNNIKLYVR 359

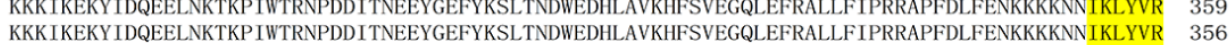
KKKIKEKYIDQEELNKTKPIWTRNPDDISNEEYGEFYKSLTNDWEDHLAVKHFSVEGQLEFRALLFIPRRAPFDLFENKKKKNNIKLYVR 357 KKKIKEKYIDQEELNKTKPIWTRNPDDITNEEYGEFYKSLTNDWEDHLAVKHFSVEGQLEFRALLFIPRRAPFDLFENKKKKNNIKLYVR 360

RVFIMDSCEELIPEYLNFVRGVVDSEDLPLNISREMLQQSKILKVIRKNIVKKCMELFGELAEDRENYNKFYDGFSKNLKLGIHEDSQNR 445 RVFIMDNCEELIPEYLNFVRGVVDSEDLPLNISREMLQQSKILKVIRKNIVKKCLELFAELAEDKENYKKFYEGFSKNIKLGIHEDSQNR 446 RVFIMDNCEELIPEYLNFVRGVVDSEDLPLNISREMLQQSKILKVIRKNIVKKCLELFAELAEDKENYKKFYEGFSKNIKLGIHEDSQNR 446 RVFIMDNCEELIPEYLNFVRGVVDSEDLPLNISREMLQQSKIFKVIRKNIVKKCLELFAELAEDKENYKKFYEAFSKNIKLGIHEDSQNR 44 RVFIMDNCEELIPEYLNFVRGVVDSEDLPLNISREMLQQSKILKVIRKNIVKKCLELFAELAEDKENYKKFYEGFSKNIKLGIHEDSQNR 449 RVFIMDNCEELIPEYLNFVRGVVDSEDLPLNISREMLQQSKILKVIRKNIVKKCLELFAELAEDKENYKKFYEAFSKNIKLGIHEDSQNR 446 RVFIMDNCEELIPEYLNF IRGVVDSEDLPLNISREMLQQSKILKVIRKNIVKKCLELFAELAEDKDNYKKFYDAFSKNLKLGIHEDSQNR 447 RVFIMDSCEELIPEYLNFI RGVVDSEDLPLNISREMLQQSKILKVIRKNIVKKCLELFAELSEDKDNYKKFYEAFSKNIKLGIHEDSQNR 450

S.salar

P.olivaceus

S.chuatsi

L.calcarifer

Tovatus

S.dumerili

C.batrachus

S.salar

P.olivaceus

S.chuatsi

L.calcarifer

Tovatus

S.dumerili

D.rerio

C.batrachus

S.salar

P.olivaceus

S.chuatsi

L.calcarifer

Tovatus

S.dumerili

D.rerio

C.batrachus

S.salar

P.olivaceus

S.chuatsi

S.chuats

L.calcarifer
Tovatus

S.dumerili

D.rerio

C.batrach
KKLSELLRYHSSQSGDELTSLTEYLTRMKDNQKSIYYITGESKDQVANSAFVERVRKRGFEVLYMTEPIDEYCVQQLKEFDGKTLVSVTK 535 KKLSELLRYQSSQSGGESTSLTEYLSRMKENQKS IYYITGESKDQVANSAFVERVRKRGFEVLYMTEPIDEYCVQQLKEFDGKTLVSVTK 536 KKLSELLRYHSSQSGDETTSLTEYLTRMKENQKSIYYITGESKDQVANSAFVERVRKRGFEVLYMTEPIDEYCVQQLKEFDGKSLVSVTK 536 KKLSELLRYHSSQSGDETTSLTEYLSRMKENQKSIYYITGESKDQVANSAFVERVRKRGFEVLYMTEPIDEYCVQQLKEFDGKSLVSVTK 536 KKLSELLRYHSSQSGDEMTSLTEYLSRMKENQKSIYYITGESKDQVANSAFVERVRKRGFEVLYMTEPIDEYCVQQLKEFDGKSLVSVTK 539 KKLSELLRYHSSQSGDETTSLTEYLSRMKENQKSIYYITGESKDQVANSAFVERVRKRGFEVLYMTEPIDEYCVQQLKEFDGKSLVSVTK 536 KKL SELLRYOSSQSGDEMTSL TEYVSRIKFNOKS IYYITCESKDQVAHSAFVERVCKRGFEVLYYTEP IDEYCVOQLKDFDGKSLVSVTK 537 KKLSELLRYHSSQSGDEMTSLAEYLGRMKENQKS IYYITGESKDQVAHSAFVERVHKRGFEVLYMVEPIDEYCVQQLKEFEGKTLVSVTK 540

EGLELPEDEEEKKKMDEDKTKFENLCKLMKEILDKKVEKVTVSNRLVSSPCCIVTSTYGWTANMERIMKAQALRDNSTMGYMMAKKHLEI 625 EGLELPEDEEEKKKMEEDKAKFENLCKLMKEILDKKVEKVTVSNRLVSSPCCIVTSTYGWTANMERIMKAQALRDNSTMGYMMAKKHLEI 626 EGLELPEDEEEKKKMEEDKAKFESLCKLMKEILDKKVEKVTVSNRLVSSPCCIVTSTYGWTANMERIMKAQALRDNSTMGYMMAKKHLEI 626 EGLELPEDEEEKKKMEEDKGKFESLFKLMKEILDKKVEKVTVSNRLVSSPCCIVTSTYGWTANMERIMKAQALRDNSTMGYMMAKKHLEI 626 EGLELPEDEEEKKKMEEDKAKFESLCKLMKEILDKKVEKVTVSNRLVSSPCCIVTSTYGWTANMERIMKAQALRDNSTMGYMMAKKHLEI 629 EGLELPEDEEEKKKUEEDKAKFESLCKLMKEILDKKVEKVTVSNRLVSSPCCIVTSTYGWTANMERIMKAOALRDNSTMGYMMAKKHLEI 626 EGL EGLELPEDEDEKKKMEEDKAKFENLCKLMKE ILDKKVEKVTVSNRLVSSPCCIVTSTYGWTANMERIMKAQALRDNSTMGYMMAKKHLEI 627

NPDHPIVETLRQKADLDKNDKAVKDLVILLFETALLSSGFSLDDPQTHSNRIYRMIKLGLGIDDDE-VIPEEPTSAPAPDEIPPLEGD-- 712 NPDHPIVETLRQKADADKNDKAVKDLVILLFETALLSSGFSLDDPQTHSNRIYRMIKLGLGIDD-DDVPTEETTSAAVPDEIPLLEGDGE 715 NPDHPIVETLRQKADSDKNDKAVKDLVILLFETALLSSGFSLDDPQTHSNRIYRMIKLGLGIDD-DDVPIEEATSTAVPDEIPPLEGDGD 715 NPDHPIVETLRQKADADKNDKAVKDLVILLFETALLSSGFSLDDPQTHSNRIYRMIKLGLGIDD-DDVPTEEATSTAVPDEIPPLEGDGD 715 NPDHPIVETLRQKADADKNDKAVKDLVILLFETALLSSGFSLDDPQTHSNRIYRMIKLGLGIDD-DDVPTEEATSTSVPDEIPPLEGDGE 718 NPDHPIVETLRQKADADKNDKAVKDLVILLFETALLSSGFSLDDPQTHSNRIYRMIKLGLGIDD-DDVPTEEATSTSVPDEIPPLEGDGE 715 NPDHPIMETLRQKAEADKNDKAVKDLVILLFETALLSSGFSLDDPQTHSNRIYRMIKLGLGIDEDEDVPVEEPSSAAAPEDIPPLEGD-- 715 NPDHPIMEMLRQKAEADKNDKAVKDLVILLFETALLSSGFSLDDPQTHSNRIYRMIKLGLGIDD-DDVPSDEPSSAPAPEEIPPLEGD-- 717

DDASRMEEVD 722

DDASRMEEVD 725

DDASRMEEVD 725

DDASRMEEVD 725

DDASRMEEVD 725

DDASRMEEVD 728

DDASRMEEVD 725

DDASRMEEVD 725

DDASRMEEVD 727 
Figure 2 Alignment of the $H S P 9 O \beta$ amino acid sequences of $T$. ovatus and other fishes. The yellow shades represent the five conserved signaling regions of the HSP9O family. The cytoplasmic HSP9O signature MEEVD is indicated by a turquoise shade. The number on the right indicates the number of amino acids.

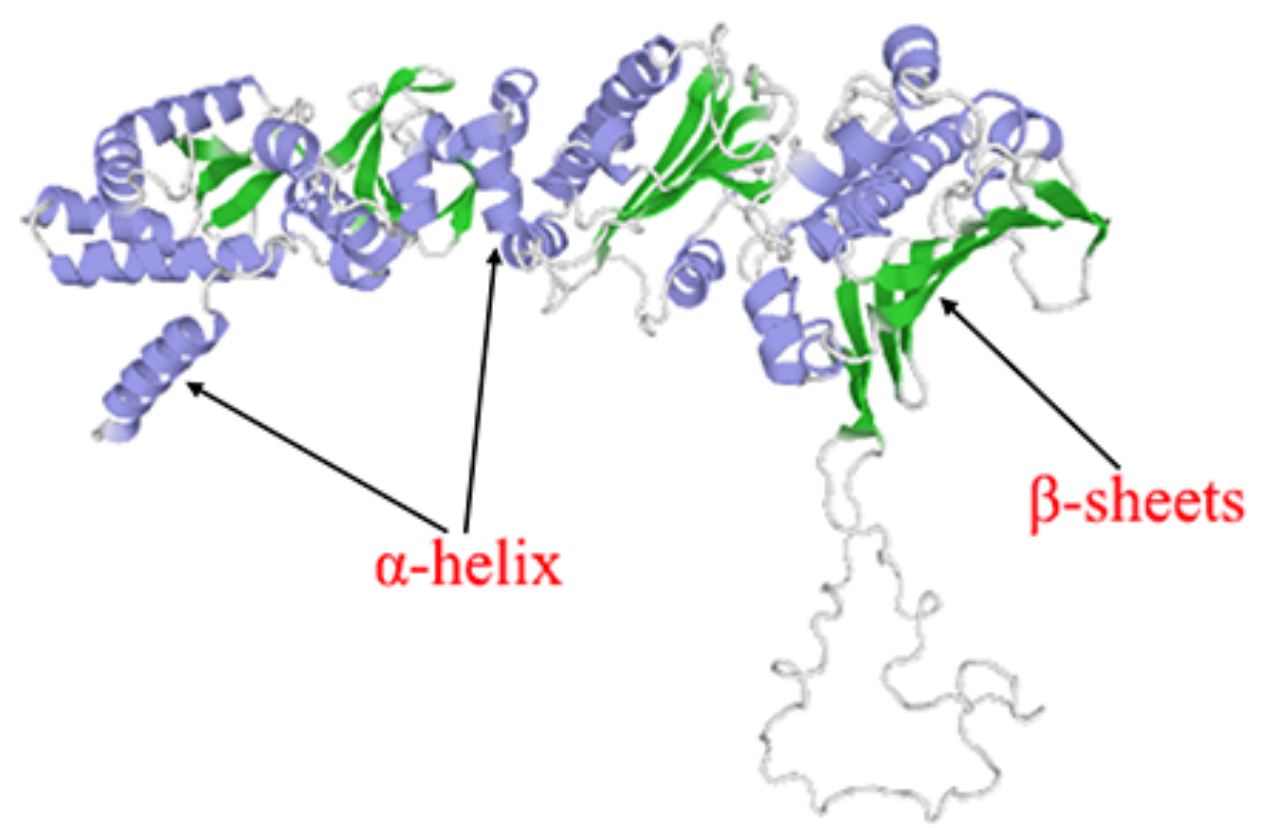

Figure 3 Three-dimensional structure model of the TOHSP9O $\beta$ gene protein of $T$. ovatus. The purple and green colors in the figure are a-helix and $\beta$-fold.

Table 2 Multiple alignments and phylogenetic tree construction of the desired species sequence.

\begin{tabular}{|c|c|c|c|c|c|}
\hline \multirow[b]{2}{*}{ Species } & \multirow[b]{2}{*}{ Order } & \multirow{2}{*}{$\begin{array}{c}\text { Gene } \\
\text { isoform }\end{array}$} & \multirow{2}{*}{$\begin{array}{l}\text { Accession No. } \\
\text { (NCBI) }\end{array}$} & \multicolumn{2}{|c|}{ Trachinotus ovatus } \\
\hline & & & & $\begin{array}{c}\text { Identity } \\
(\%)\end{array}$ & $\begin{array}{l}\text { Pairwise } \\
\text { distance }\end{array}$ \\
\hline Trachinotus ovatus & Perciformes & HSP90ß & MN641479 & 100.00 & 0 \\
\hline Seriola dumerili & Perciformes & HSP9Oß & XP_022619895.1 & 99.17 & 0.008 \\
\hline Echeneis naucrates & Perciformes & HSP9Oß & XP_029351674.1 & 98.90 & 0.011 \\
\hline Siniperca chuatsi & Perciformes & HSP90ß & AJP74821.1 & 98.21 & 0.015 \\
\hline Stegastes partitus & Perciformes & HSP90ß & XP_008274978.1 & 98.07 & 0.017 \\
\hline Lates calcarifer & Perciformes & HSP90ß & $\mathrm{AEH} 27541.1$ & 98.07 & 0.018 \\
\hline Collichthys Iucidus & Perciformes & HSP90ß & TKS76250.1 & 97.79 & 0.018 \\
\hline Salmo salar & Salmoniformes & HSP90ß & AAD30275.1 & 92.51 & 0.074 \\
\hline Paralichthys olivaceus & pleuronectiformes & HSP9Oß & ABG56394.1 & 97.10 & 0.03 \\
\hline Danio rerio & Cypriniformes & HSP90ß & NP_571385.2 & 94.06 & 0.054 \\
\hline Clarias batrachus & Siluriformes & HSP90 & AGI03840.2 & 94.63 & 0.051 \\
\hline Numida Meleagris & Galliformes & HSP90ß & XP_021245225.1 & 91.11 & 0.086 \\
\hline Corapipo altera & Passeriformes & HSP9Oß & XP_027497163.1 & 90.56 & 0.092 \\
\hline Ophiophagus Hannah & Squamata & HSP90ß & ETE64918.1 & 89.47 & 0.103 \\
\hline Homo sapiens & Primates & HSP9Oß & NP_031381.2 & 91.11 & 0.087 \\
\hline Pongo abelii & Primates & HSP90ß & NP_001126444.1 & 91.11 & 0.087 \\
\hline Equus caballus & Perissodactyla & HSP90ß & NP_001075407.1 & 91.25 & 0.086 \\
\hline Cavia porcellus & Rodentia & HSP90 & XP_003473881.1 & 90.97 & 0.089 \\
\hline Xenopus tropicalis & Anura & HSP90 $\beta$ & NP_001025655.1 & 91.52 & 0.081 \\
\hline
\end{tabular}


The phylogenetic trees of each species were constructed by neighbor-joining (NJ) method of MEGA6.0 (Figure 4), and the analysis showed that amphibia, aves, reptiles, mammals and osteichthyes were clustered into one branch respectively. Perciformes are grouped as a single branch in osteichthyes, and $T$. ovatus forms a small cluster with $S$. dumerili, E. naucrates and L. calcarifer, and has the highest correlation with HSP9O $\beta$ evolution of $S$. dumerili. Therefore, it can be determined that the ToHSP9O $\beta$ should belong to the cytosolic HSP90ß subfamily.

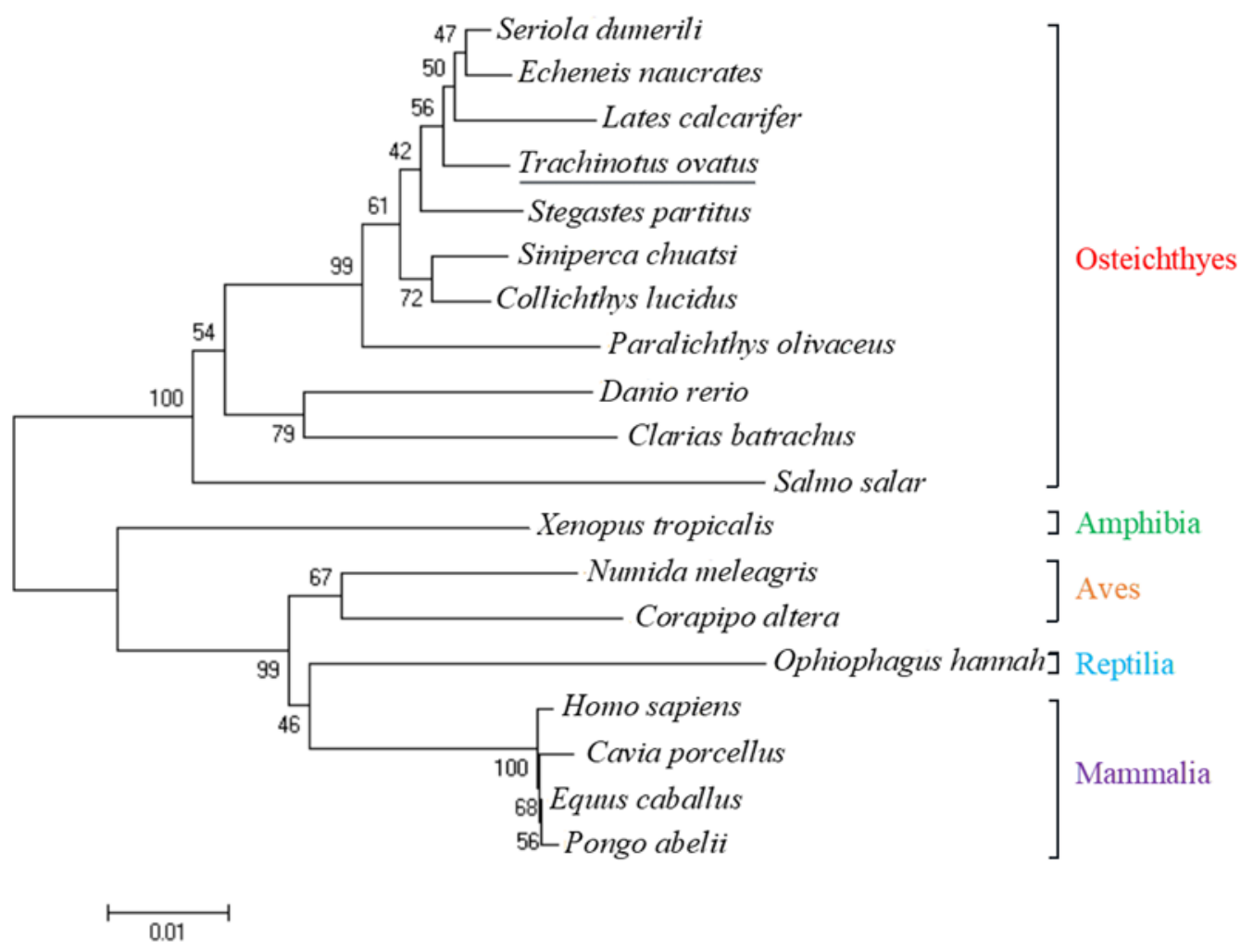

Figure 4 Neighbor-joining (NJ) phylogenetic tree constructed based on amino acid sequence encoded by the ToHSP9Oß gene.

Genomic structures analysis of ToHSP9OB. The genomic sequences of ToHSP90 $\beta$ (GenBank accession number: MN708358) were amplified and confirmed (Figure 5). We found that the ToHSP90 $\beta$ genomic sequences length is $6946 \mathrm{bp}$, containing 12 exons and 11 introns, and all the introns and exons boundary sequences follow the GT-AG rule. The first exon (130bp) is an untranslated region, the last exon consists of a coding sequences (116bp) and a non-coding sequences (334bp), and the other exons are translation regions. Comparison of exons and introns structures of HSP90 $\beta$ genomic sequences of different species indicated that their HSP90 $\beta$ genomic structures containing 12 exons and 11 introns (Figure 6). The genomic structures of $T$. ovatus ToHSP9O $\beta$ is highly similar to $S$. dumerili. The exons differences of all species are mainly concentrated in the first and twelfth, and the other exons are basically the same, therefore, the protein encoded by this gene is highly conserved. The length of introns larger difference.

Expression pattern of ToHSP9O $\beta$ in different tissues. The tissue expression characteristics of ToHSP90 $\beta$ mRNA in healthy $T$. ovatus was analyzed by qRT-PCR is shown in Figure 7. The results showed that the relative expression of ToHSP90 $\beta$ mRNA was highest in the intestine tissue compared to other tissues $(P<0.05)$. Secondly, the expression levels of this gene were relatively high in the kidney, gill, fin, and liver, and were relatively low in the spleen, brain, muscle, and blood. 
1 etgaaacgeatgtgtataaagatteeggtetattaaaaggaagtecettecattecetgegteagagageagaagggacageacattggttgeatttatteaagaaaagteaacaca 121 aataaacaaggthatgtacacgagtgttteettgegaataaageacattgttetecgttaatattggagttt gecaacecgtetetgaattattecaacggatgtatecatgagagetc 241 aagagecacaeggtaacettageatcateactgtgtgeggetacagttageteattaatgttatcacttatetgtetgeatacatgttaatgataacgttaateettaaategtga

361 gattgtegtgtategatggacggtgtgtggtgetgageggtgtaaatgeggtactectgecgcactgetaggecaggeceggettgggttgeaceaaggcaggtgtggtggagatgacaa

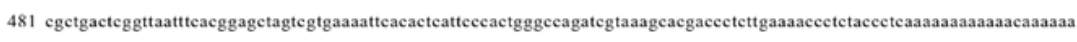
601 caaaaacgtgttegtgtaatatceatttatgtaacgattacttgeagttgttggtgategtaacggecegttaagttacgtaaccaacggaaaacaacggettggtteatgttaacg 721 tecgtgtgtgttcagatteatteggttattgeagttgthtegetaacggecagtgattggttggttegtettgatageggagacaaagetgettgttgtacegteaggttggt 841 cetagtegttgteatatgtggetttaactectacttattaacgtttgeteacaagteacegtgtagtgeegeatttecggegttgtegttatttaateacgggetgetegtaggea 961 egtgttgeggagtecattgtetgtaggtegacgecatgttggttaacggetacttecgeegatatgtagtaacacgatggetgeggtecagtettaggaaaceggttgtggeettgga 1081 teaaattattategegtttacgattaacgaaactgaatgectacaatetgteattaacacacaagagectteaacttetgtggeagtectettegggttgegtteagttragtteate 1201 acecetgataatgtacatgtgtttttaatttgtactgttcaaacecaaageaatttgetaaaacattatcegatttgtgtttgtge日gatgectgaagaaatgeaceaagaggagga 1321 ggetgagacetttgecttceaggeagagattgetcagetgatgtecetgatcatcaacacettetactecaacaaagagatettectcagggagttgatetetaatgettetgatgthaa 1441 tetteaggagttggacttgtttttettgttacaceataagtaaaatgattteaagtgectetgttatgatgaggtatttaataatcaagttaataatataacatgatgtgtattttg 1561 cttatgtgtgttaceggetttggacaaaatecgetatgaaagectgactgacecttceaagetggacageggeaaggatetgaaaattgacateatececaacaaagetgacegtacec 1681 tgacectcategacactggaatcggeatgaccaaagetgacetgattaacaacetgggtaceatcgecaagtetggcactaaggectteatggaggecetgcaggtheaacgaaacatga 1801 tecagattgeagcatgtggaagatgaagecaaatggeacataagacaagagcaettetatctaaceatgeattgtcaaaggettaaattgeaacacaaagageagtgataatacattge 1921 cttcagagttattaaaggat getceagactgtttgaatgecatattaagttcaaataattgtgttecettrag getggagetgacatetecatgattggtcagtttggtgtgggtttc 2041 tactetgectacettgtggetgagagggtggttgteatcaccaaacacaacgatgatgageagtatgectgggagtectetgetggeggtteettcacegteaaggttgacaacg gttcg 2161 tgteceggeatcattacaatatcaaageagetgagatggeaaegtttgacataatgtgecaattgttetgaceattttgacactgeacttcaaaagttgacatgetegteceatattc 2281 atcatgttggtataagagetgeaatgatcaacggecagttgagaaattaaaggaccagetattggagaattgttaagtaattetttecaagetcaaacatgagtattggaactget 2401 teettggttagteatgtgtagtaaactgagttcageetgeacaagacatttaagatgteacttgtgtttgagtteaaggececacagteaaataattgaaaataatggaccac 2521 ttattetaatgtaaatattggttagtegtagecetggttgtatettgeacettgttacttattcaactaactgeagttettgtteaaagettaaacaggaaceggagaaaacaat 2641 tattutttaatattggaettgaacageactgttaataacagtatetaattagtggtgattacaatgtaaatgatgaaatatgaageettggtggteatatggetagtagag 2761 ggtagetgetecatettecaacaagtgegaatggetgtacaacttgteaac日g gtgageceattggecgtggaacaaagategtettgtaectgaaggaggaceagacagagtacattg 2881 aggagaagagggtcaaggagattg taaagaageactcteagttcatcggetaceceatcacectgtt gtaagtatttagtgttecaettcetttagttcagteaggtttactgttgc

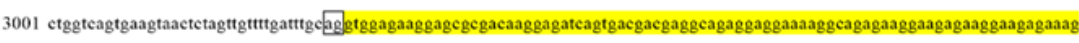
3121 aggacggtgaggacaagecaaagattgaggatgtgggetcagatgatgaggaggactecaaagacaaggacaagaagaagaaaaagaagatcaaggagaagtacattgaceaggaggage 3241 tgaacaagaceaageceatetggaccagaaacectgatgacatcacaaacgaggaatatggegagttctacaagagtetgaceaatgactgggaggatcacetegetgteaag gtaaca 3361 tgaaaggetaaageaactgttgcattacatatgettcagecagetgggtgectaaatgtaatggtaaattaataaattettececttcacegeatteteagtggagggecaget 3481 tgagttecgegecetgetetteattececgtegtgeacetttgacetettgagaacaagaaaagaagaataacatcaagetgtacgtgaggagagtettateatggacaactgtga 3601 agaacteateceagagtacetgag thagttggtgecttecectgggattatagaatatteteteagetetgeactgactattgtetataactacaaageattaatataatactgeaa 3721 eatgtecaactaaacegtgatttg taaattttttttaatttttttttte tegacttgtecgtggtgtggtggacteagaggacetgececteaacateteaagagaaatgetge 3841 agcagageaagatecteaaggteattegeaagaacattgteaagaagtgtetggagetettgetgaactggetgaggataaggagaattacaagaagttetatgaaggattetecaaga 3961 acateaaggtateaaastaaacttettgtgetceatattgttttaaattacacaaatettttaacaagetttaateaaagttectectecatattgeattetgtgtgtetteagtgo 4081 atetceatggattcataaggtteacectcattgttgtggtgetgetgttecageettaaacgeagtgtagteatagttagtttgetteggtcagetteagacacagetgaagaaa 4201 gttgttattuttetgaatteteaateaaetcaaatggtatgtaatggageatcagetagtgeacagaaettgtaettacaagtettatgtgetgtagactagagteatgtagegetg 4321 tagattagteagteaattagtattgttaaattggtaactagacattcacecagttaccetaaaatattacttgectgtgacatggtgteacatgecaacacaatcagettgtectactgt 4441 atgectaattaacagaaaagtaaagtateattgeatagteagetetggggectgtataaagaageaagttaaettggaetetetgettaectggetteactgageeteacetgtgc 4561 agtectgtgtaaacattaagaagetgggettaacaatgaagetatgagtgtatteatgtgaaatgatttgaacagggttgteatetaategtgactttgtetttatgtttaaatggag 4681 ceatettgagttteaaaateetgagttaaacetggagtgtaggtggggaattggttagecteacttgatacatttgggtgtetttgagtttgaacaattgtecattategtgtc

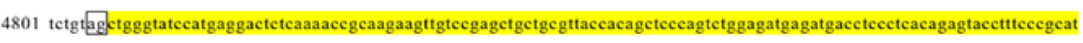
4921 gaaggaaaaceagaagtecatetactacattactg国gagttgectagaatattgattugtgatagtetgtaaaacttcagtgigtgiggggggggggtgtttaagaaaattgtat 5041 gtatcaagataagtaaaactaatgetttatttaaageaaaceatattaatetgecagaataatactgatectcatattgttatatetetgggtgagageaaggateaggtggecaact 5161 cegetttgtegagegtgtecgeaagegtggettgaggtectgtacatgacagageceattgatgagtactgtgtecageagttgaaggagttgatggeaagagettggtetetgtea 5281 ceaaagagggectggagetgecagaggatgaggaggagaagaaaaagatggaggaggacaaggecaagttegagagectetgeaageteatgaaggagatcettgacaagaaagtggaga 5401 ag 2 teagtgatcettatgaaetgtttg tattettactatatttcetteataatgeaggatttaggeccataaagttcacaactgeattgacagtgett gtttggtaacaggtgtec 5521 taatteateceaacttgtattgtggtgagtteatgeagacttggtetecttaggtttetgtgtteaagegtacgttgaatgattgeagttttectaaatgttgacaagtaagggt 5641 tecagatgatgtgtggagacgetgagagtccagaacecttgaaaaacetaactgattgtaatactggtetaacatatgectgagttcagtttgeatacttggatttatagaatgg 5761 aagttacaacttetgaaattggtgagegettaaccaattgtttatgtatgtattggecactgtgttaacatgaaggtetacagaccagatattgaaggtacegtagaactggtaaaca 5881 aatggttgecettgacatgetggtgtgttattectaacaagactattetgeatgtgttuttgaatteaceecgtettgaatuttatgtaggetaacetgetgttggacactatgea 6001 tgttgtgggttutttgtttggteceececececaaaagtettaatgtecetettggetgteteatec gggtgacagtgtecaacagactggtgtetteacect getgeattgtg 6121 acaagtacttacggetggacggecaacatggagaggatcatgaaggeccaggeactcagggacaactccaccatgggetacatgatggecaagaagcacetggagatcaaccetgaccac 6241 cecategtggagaeteteaggeagaaggecgacgetgacaagaacgacaaggetgtgaaggacetegteatectgetettegaaacegecetgetgtecteaggettetecttggacgac 6361 ecacagacecactecaacegeatetacagaatgatcaaacttgggetgg国gaggacgeactgeagataaacttgaaacttaggacactagtgettgtttgatettaacaaataaca 6481 attettgtaceteteggtategatgacgacgatgtteceacagaggaggecaettetacatecgteceagatgagatteetecectagaaggegatggtgaagatgacgettecegtatg 6601 gaagaagttgattaaatetaeceacecectgatttetaacacttagecteacttteaattgtteatecttaaaactgeagtaactgeaaaacaaatagteatteatgttgtgtggtg 6721 gaceagtgttgetettgtgtetagageattactetgeaagacetttaagaaageagtttggttt getgtaataagtteatggtgacageacattgtttaacaagtacetgtt 6841 geactgagtttaaatgttggagtggtaaatgtgtgaacatgggaatggtacattecattatcaggtetggagggttgggaggttetgeteatgtgeaacactgo

The Israeli Journal of Aquaculture - Bamidgeh • IJA.73.2021.1531108 
Figure 5 The genomic sequence of ToHSP90 3 . The yellow shaded portion indicates the exon sequence. The two special sites (GT-AG rule) for each intron splicing are represented by a rectangular box.

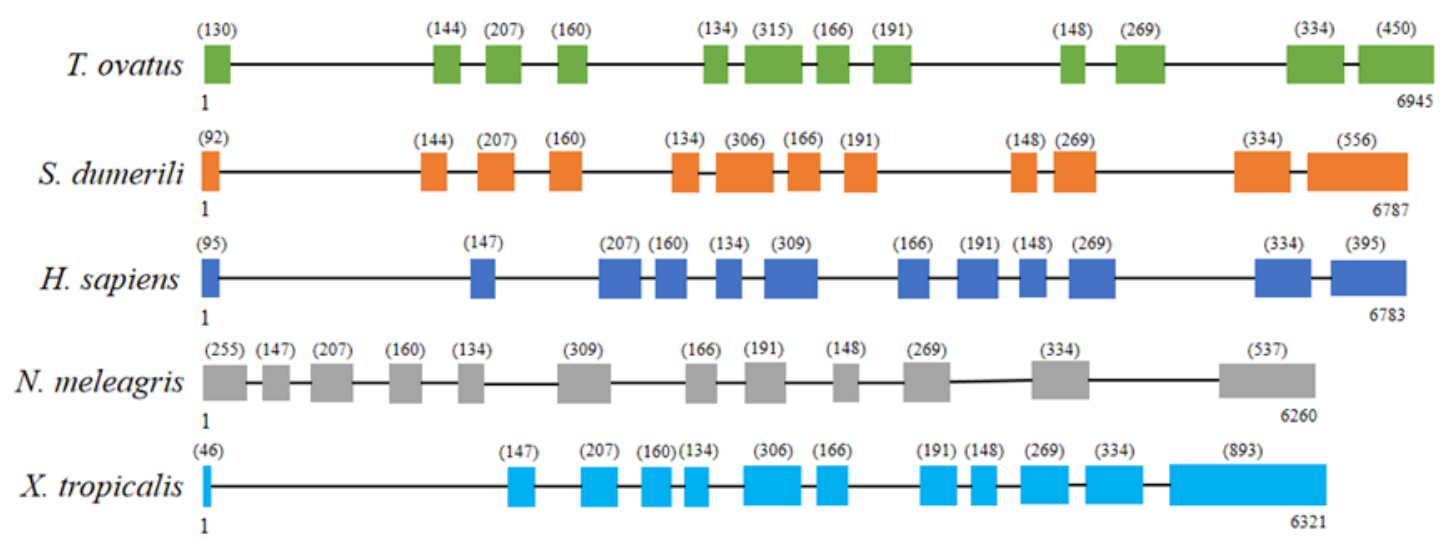

Figure 6 Comparison of the structure of introns and exons of the ToHSP9O $\beta$ gene with other species. Black lines and rectangles represent introns and exons, respectively. The numbers in parentheses in the figure indicate the number of exon base pairs. The accession number of HSP9O $\beta$ gene sequence of the species in GenBank: S. dumerili (NW_019174280.1), H. sapiens (NC_000006.12), N. Meleagris (NC_034411.1), X. tropicalis (NW_016684028.1).

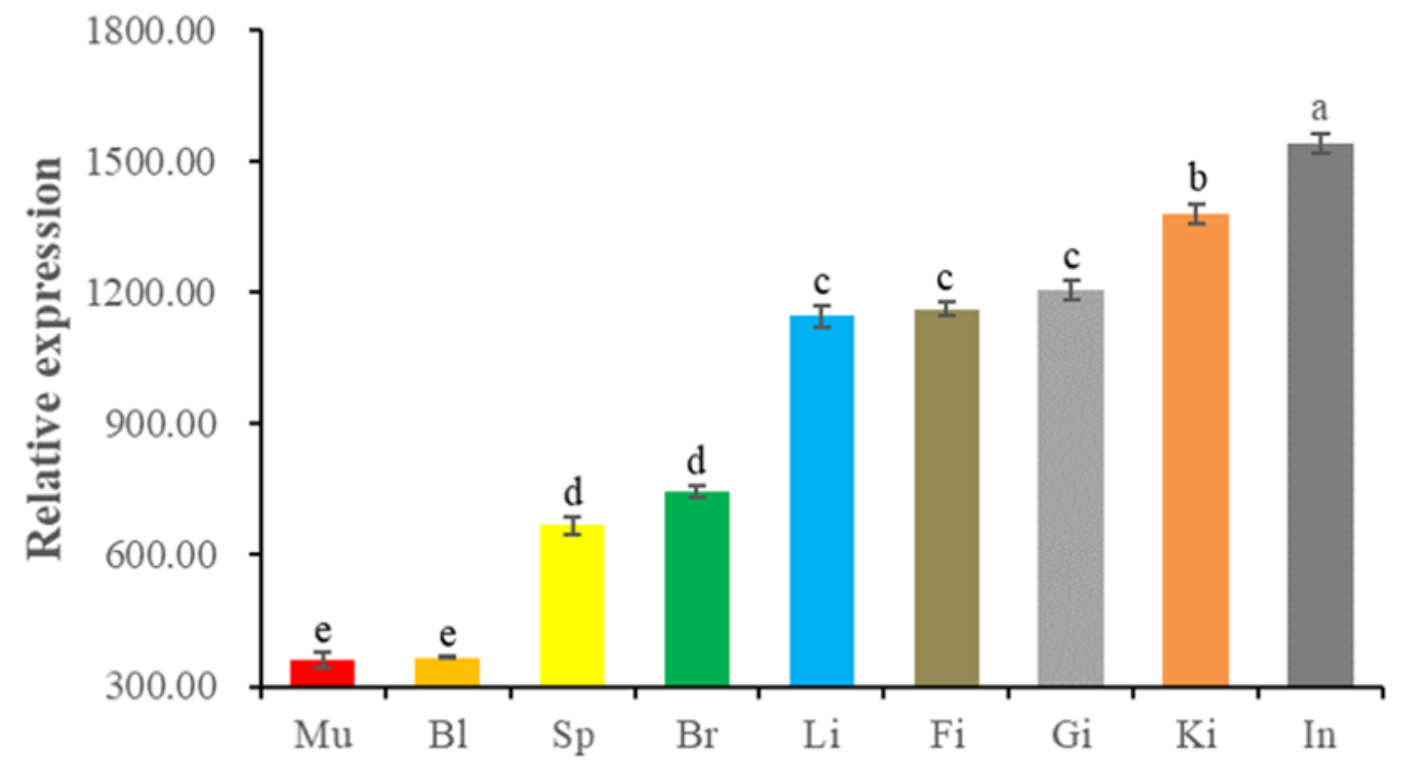

Figure 7 Tissues expression characteristics of the ToHSP90 $\beta$ mRNA in healthy $T$. ovatus. Different letters indicate significant difference between groups $(P<0.05)$. Vertical bars denote standard error. $\mathrm{Mu}$, muscle; $\mathrm{Bl}$, blood; Sp, spleen; Br, brain; Li, liver; Fi, fin; Gi, gill; Ki, kidney; In, intestine.

Expression levels of ToHSP9O $\mathrm{B}$ RNA at the density stress and C. irritans infection. The differential expression of ToHSP90 $\beta$ mRNA in liver, kidney, brain, and muscle of $T$. ovatus under density stress by qRT-PCR is shown in Figure 8. The results showed that the relative expression levels of ToHSP90 $\beta$ mRNA in the kidney and brain were significantly upregulated as increasing stocking density $(P<0.05)$. However, the relative expression of ToHSP90 $\beta$ mRNA in the liver was not statistically significant among groups $(P>0.05)$. The relative expression levels of ToHSP90 $\beta$ mRNA in the LD and HD groups were significantly higher than those in the MD group in muscle $(P<0.05)$. The differential expression of ToHSP90 $\beta$ mRNA in liver, kidney, and gill of $T$. ovatus after $C$. irritans infection by qRT$P C R$ is shown Figure 9. 

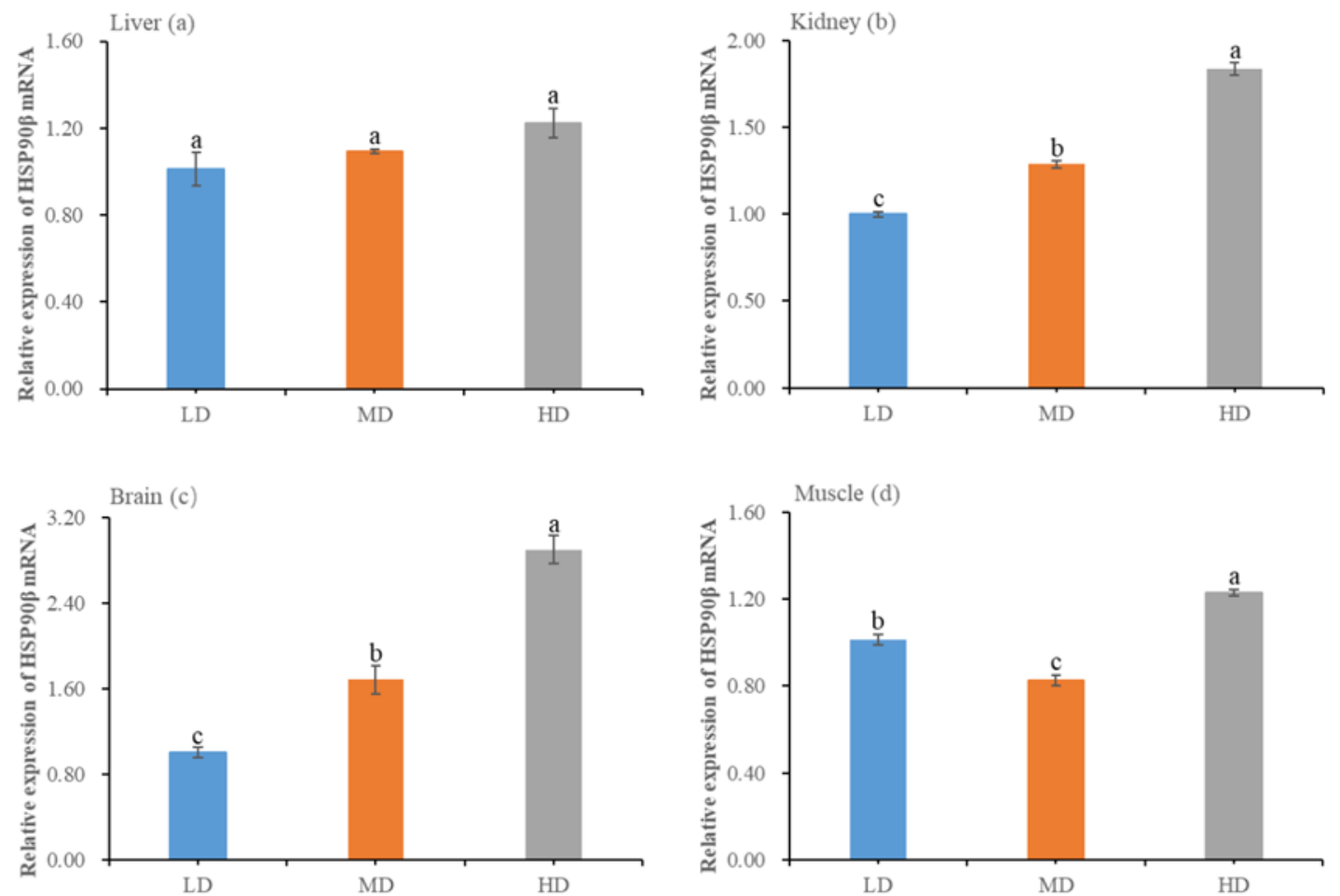

Figure 8 Relative expression levels of ToHSP90 $\beta$ mRNA in the liver (a), kidney (b), brain (c) and muscle (d) of juvenile golden pompano cultured at different stocking densities for 10 weeks. Data are expressed as the mean \pm SE $(n=3$ per cage). Different letters indicate significant differences between groups $(P<0.05)$. Vertical bars denote standard error. LD, low stocking density; MD, medium stocking density; HD, high stocking density.
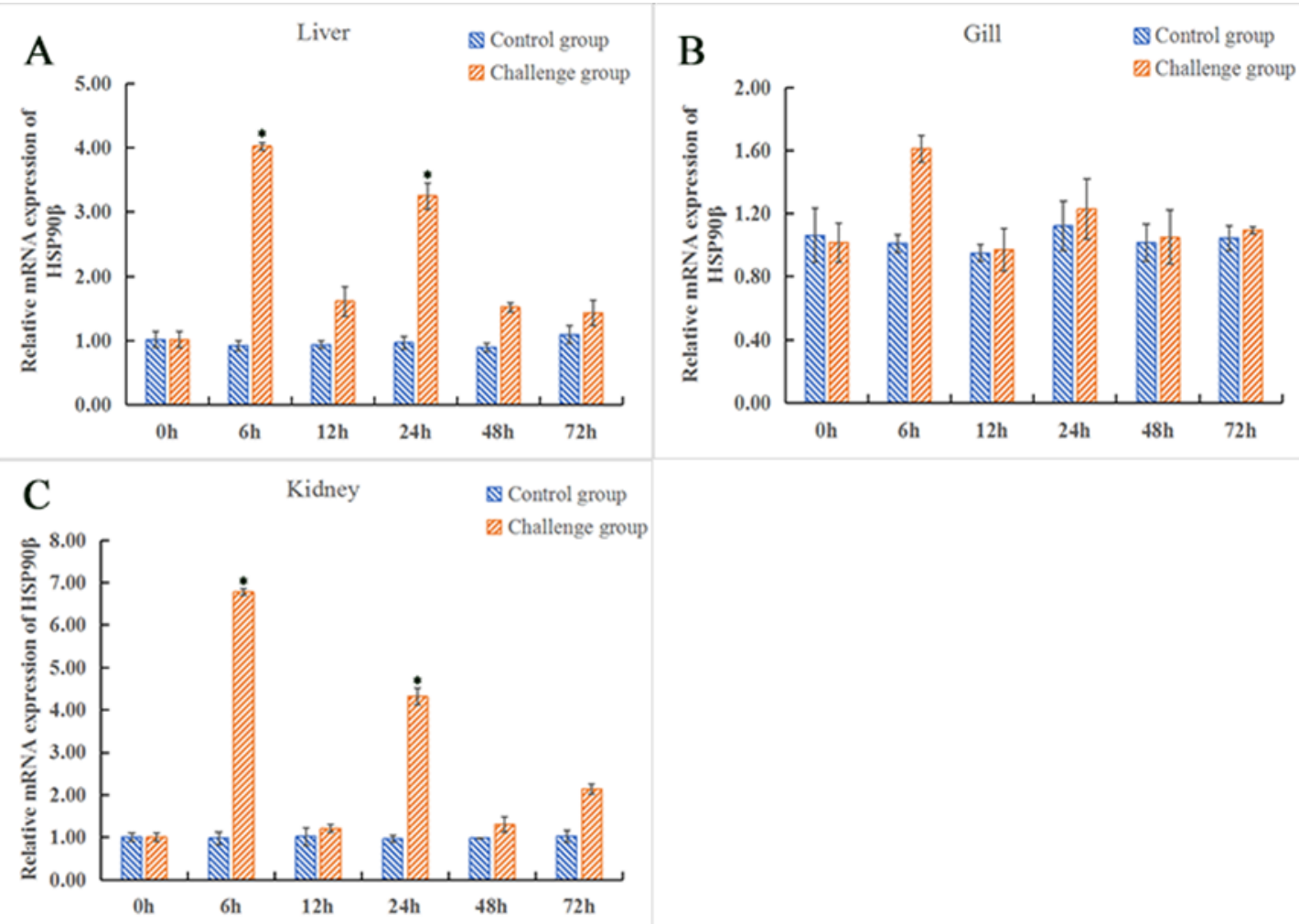

Figure 9 Relative expression levels of ToHSP90 $\beta$ mRNA in the liver (a), kidney (b) and gill (c) of juvenile golden pompano cultured after Cryptocaryon irritans infection. Data are expressed as the mean \pm SE $(n=3$ per cage). Different letters indicate significant differences between groups $(P<$ 
0.05). Vertical bars denote standard error.

\section{Discussion}

Heat shock protein 90 is a class highly conserved protein and important molecular chaperone, and plays a role in enhancing animal adaptability to environmental stress (Zhang et al., 2009). In this study, the complete cDNA sequence of ToHSP90 $\beta$ gene was obtained for the first time, with a total length of $2648 \mathrm{bp}$. The ORF of $2184 \mathrm{bp}$ can encode a protein of 728 amino acids. Analysis indicated that it has the Heat shock proteins 90 family signature (YSNKEIFLRE) (Mo et al., 2018). The ToHSP90 $\beta$ has a histidine kinase-like ATPases domain (Figure 1), which is the major structural and functional domains in HSP90 family, this region is highly conserved and has the activity of hydrolyzing ATP (Wei et al., 2013). Multiple sequence analysis of the ToHSP90 $\beta$ amino acid revealed that it has five conserved signal regions of the HSP90 family with other fish in Figure 2 (Gupta, 1995). The C-terminus of HSP90 $\beta$ has a conserved MEEVD structure that is a feature shared in all the cytosolic Heat shock protein 90 family (Scheufler et al., 2000; Qin et al., 2016; Zhou et al., 2017; Rahimi et al., 2018). Scheufler et al. (2000) suggested that MEEVD can be recognized by the TPR domain of HOP (HSP70 and HSP90 organizing protein) and form a molecular chaperone complex with heat shock protein 70. From the above, it is known that the HSP90 gene is highly conserved during evolution.

Amino acid homology analysis showed that the amino acid sequence of ToHSP90 $\beta$ of $T$. ovatus has higher homology with other known fishes (92.5-99.17\%) and the highest homology with S. dumerili (99.17\%). Wei et al. (2013) showed that Miichthys miiuy HSP90 has $91.7 \%-99.5 \%$ homology with other species, especially the large yellow croaker HSP90 $\beta$ amino acid sequence is as high as $99.5 \%$, which is similar to our results. The Poisson Correction distances of the HSP9O $\beta$ amino acids of $T$. ovatus and other osteichthyes teleost fish are 0.008-0.074, respectively. The closest distance between $T$. ovatus and $S$. dumerili is 0.008 . Phylogenetic tree results showed that $T$. ovatus scorpion formed a small cluster with S. dumerili, E. naucrates and L. calcarifer, and had the highest correlation with $S$. dumerili evolution. In summary, the ToHSP90 $\beta$ is highly consistent with HSP9O $\beta$ of other species, and it is indicated that ToHSP90 $\beta$ should belong to the HSP90 $\beta$ subfamily.

The expression analysis of $T$. ovatus ToHSP90 $\beta$ showed that it had different degrees of expression in the nine tissues examined, and the highest expression in the intestine, followed by the kidney, gill, fin and liver. Generally, intestine, kidney, and liver of fish are regarded as immune organs and central to the immune responses (Alvarez-Pellitero.2008). Qin et al. (2016) showed that the HSP90 $\beta$ mRNA of $B$. reevesae was expressed in the tissues studied, and the expression was significantly higher in the skin and kidney. Song et al. (2018) showed that the HSP9Oß mRNA of M. amblycephala has the highest expression level in the hindgut, and its expression level was different between tissues, indicating that it may play a protective role through tissue-specific mechanisms. Wei et al. (2013) showed that the highest expression of HSP90 $\beta$ mRNA was observed in the liver of M. miiuy, which was specifically expressed between tissues, which may be caused by different protein synthesis in different tissues. Different expression patterns of HSP9O $\beta$ in fish indicate that it may be involved in various physiological processes of fish.

The environmental changes cause the aquatic animals to respond to stress and cause changes in the body's physiological mechanisms and biochemical reactions. Stocking density, an important environmental stress factor and is also one of the important factors affecting fish welfare (North et al., 2006; Calabrese et al., 2017). To maintain the homeostasis of the body, adaptive physiological responses occur in the aquatic animal body to cope with adverse environmental stresses such as the HSP90 family of "molecular chaperone" is involved (Zhang et al., 2009; Lin et al., 2018). Studies have shown increased expression of stress-related genes in the brain of $D$. labrax at high stocking densities such as HSP90 (Gornati et al., 2004). Ni et al. (2014) showed that HSP90 expression levels increased significantly in the liver and spleen of Amur sturgeon under high stocking density. However, studies have shown that stocking density has no significant effect on HSP90 mRNA expression (Salas-Leiton et al., 2010; Long et al., 2019). The reason for this 
difference may be due to differences in the species themselves or different sampling time points. Although the above study revealed the expression pattern of HSP90 mRNA under density stress, it did not indicate which subtype of gene was used. In this study, the expression of HSP9O $\beta$ in the kidneys and brain tissues of $T$. ovatus under high stocking density stress was significantly up-regulated, indicating that HSP90 $\beta$ may be involved in the physiological process of T. ovatus against chronic stress. Similarly, Kim et al. (2013) found that the expression of HSP90 $\beta$ gene in the liver and brain of $T$. obscurus was significantly up-regulated under high stocking density stress, indicating that HSP90 $\beta$ can be used as a potential indicator to evaluate fish stress. Valenzuela et al. (2019) showed that the expression of HSP9O $\beta$ in skeletal and head kidney tissues of $P$. adspersus was upregulated under high-density stress. Then, our results show that the relative expression of ToHSP90 $\beta$ mRNA in the liver was not statistical significance among groups. This may be related to the protective role of fish tissue- or time-specific mechanisms (Song et al., 2018). In summary, the expression of HSP90 $\beta$ mRNA in fish is closely related to the chronic stress of stocking density which may be that chronic stress causes protein damage in the body, thus inducing the increase of HSP9O $\beta$ expression. But the specific mechanism of HSP9O $\beta$ needs to be further studied.

In recent years, farmed fish has been strongly affected by various diseases and the mortality rate is directly related to pathogen infection (Yang et al., 2020). Studies on fish have shown that disease factors, such as bacterium, parasite and fungus could induce HSP expression (Ding et al., 2013; Song et al., 2018; Cheng et al., 2019). HHS90 can recognize pathogen-associated molecular pattern and participate in the host's innate immune response. HSP90 play important roles in resisting stress and defending against cellular pathogen infection (Ding et al., 2013; Wei et al., 2013). C. irritans is also called "white spot disease" in marine fish (Bai et al., 2020; Zhong et al., 2020). Under artificial breeding conditions, except for Chondrichthyes and Siganus that have certain resistance to $C$. irritans (Jiang et al., 2019). C. irritans has no specificity in host selection and it is pathogenic to almost all culture marine fish. Yang et al. (2020) showed that the expression of HSP90 was significantly increased in liver and kidney after C. irritans infection, suggesting that HSP90 would be involved in the immune response. Study have shown that the expression level of HSP90 in the skin of Siganus orami was significantly up-regulated $12 \mathrm{~h}$ after infection with $C$. irritans, which indicated that $C$. irritans infection triggered the immune response of skin cells (Jiang et al., 2019). Therefore, the upregulation of HSPs may be a manifestation of immune enhancement. The mRNA expression of ToHSP90 $\beta$ in liver and kidney tissues was significantly increased at $6 \mathrm{~h}$ or $12 \mathrm{~h}$ after infection, but there was no difference in gill tissue. In this study, the expressions of ToHSP90 $\beta$ gene were significantly upregulated, which may be due to their involvement in the adaptive immunomodulatory response of $T$. ovatus.

\section{Conclusion}

The full-length CDNA sequence of ToHSP90 $\beta$ gene in T. ovatus was obtained and its molecular characteristics were analyzed. The amino acid sequence of ToHSP90 $\beta$ has high homology with the amino acid sequence of other teleosts. This study was the first to analyze the expression pattern of ToHSP90 $\beta$ mRNA in $T$. ovatus tissues and their expression responses to stocking density stress and $C$. irritans infection. This gene has varying degrees of expression in the tissues studied, with the highest expression in the intestine, followed by kidney, gill, fin and liver. The results showed that the expression level of ToHSP90 $\beta$ mRNA in kidney, brain and muscle tissues was significantly correlated with the stocking densities. The mRNA expression of ToHSP90 $\beta$ was significantly increased in liver and kidney tissues, but there was no difference in gill tissue after $C$. irritans infection. Those results indicated that it has an important regulatory role in the process in responses to stocking density stress and immune regulatory response process in responses to C. irritans infection of $T$. ovatus. 


\section{Acknowledgements}

This work was supported by the National Key Research and Development Program of China (2018YFD0900301), National Natural Science Foundation Regional Innovation and Development Joint Fund (U20A2064), the China Agriculture Research System of MOF and MARA (CARS-47), Central Public-interest Scientific Institution Basal Research Fund CAFS (NO.2020TD29), Financial Fund of Ministry of Agriculture and Rural affairs of China (NHYYSWZZZYKZX2020), Key Special Project for Introduced Talents Team of Southern Marine Science and Engineering Guangdong Laboratory (Guangzhou) (GML2019ZD0605), the National Infrastructure of Fishery Germplasm Resources Project (2019DKA30470) and the Guangdong Provincial Science and Technology Project (2019B030316030).

\section{References}

Bai HQ, Zhou T, Zhao J, Chen BH, Pu F, Bai YL, Wu YD, Chen L, Shi Y, Ke QZ, Yu $\mathbf{X K}$, Xu P, 2020. Transcriptome analysis reveals the temporal gene expression patterns in skin of large yellow croaker (Larimichthys crocea) in response to Cryptocaryon irritans infection. Fish Shellfish Immunol, 99: 462-472. https://doi.org/10.1016/j.fsi.2020.02.024. Calabrese S, Nilsen TO, Kolarevic J, Ebbesson LOE, Pedrosa C, Fivelstad S, Hosfeld C, Stefansson So, Terjesen BF, Takle H, Martins CIM, Sveier H, Mathisen F, Imsland AK, Handeland SO, 2017. Stocking density limits for post-smolt Atlantic salmon (Salmo salar L.) with emphasis on production performance and welfare. Aquaculture, 468: 363-370. https://doi.org/10.1016/j.aquaculture.2016.10.041.

Chen YM, Kuo CE, Wang TY, Shie PS, Wang WC, Huang SL, Tsai TJ, Chen PP, Chen JC, Chen TY, 2010. Cloning of an orange-spotted grouper Epinephelus coioides heat shock protein 90AB (HSP90AB) and characterization of its expression in response to nodavirus. Fish Shellfish Immunol, 28: 895-904. https://doi.org/10.1016/j.fsi.2010.02.004.

Cheng J, Li HH, Huang ZY, Zhang FL, Bao LS, Li YL, Chen L, Xue LY, Chu WY, Zhang JS, 2019. Expression analysis of the heat shock protein genes and cellular reaction in dojo loach (Misgurnus anguillicaudatus) under the different pathogenic invasion. Fish Shellfish Immunol, 95: 506-513. https://doi.org/10.1016/j.fsi.2019.10.073.

Csermely P, Schnaider T, Soti C, Prohászka Z, Nardai G, 1998. The 90-kda molecular chaperone family: structure, function, and clinical applications. a comprehensive review. Pharmacol Ther, 79: 129-168. https://doi.org/10.1016/S0163-7258(98)00013-8.

Deane EE, Kelly SP, Luk JCY, Woo NYS, 2002. Chronic salinity adaptation modulates hepatic heat shock protein and insulin-like growth factor I expression in black sea bream. Mar Biotechnol, 4: 193-205. https://doi.org/10.1007/pl00021690.

Ding ZJ, Wu JJ, Su LN, Zhou FJ, Zhao XH, Deng W, Zhang J, Liu SK, Wang WM, Liu $\mathbf{H}, 2013$. Expression of heat shock protein 90 genes during early development and infection in Megalobrama amblycephala and evidence for adaptive evolution in teleost. Dev Comp Immunol, 41: 683-693. https://doi.org/10.1016/j.dci.2013.08.007.

Du C, Han YL, Hou CC, Zhu JQ, 2019. Expression pattern of heat shock protein 90AB (HSP90AB) and stress-inducible protein 1 (Stip1) during spermatogenesis of mudskipper Boleophthalmus pectinirostris. Comp Biochem Physiol, 231: 42-51. https://doi.org/10.1016/j.cbpb.2019.01.016.

Fu D, Chen J, Zhang Y, Yu Z, 2011. Cloning and expression of a heat shock protein (HSP) 90 gene in the haemocytes of Crassostrea hongkongensis under osmotic stress and bacterial challenge. Fish Shellfish Immunol, 31: 118-125. https://doi.org/10.1016/j.fsi.2011.04.011.

Gao Q, Zhao J, Song L, Qiu L, Yu Y, Zhang H, Ni D, 2008. Molecular cloning, characterization and expression of heat shock protein 90 gene in the haemocytes of bay scallop Argopecten irradians. Fish Shellfish Immunol, 24: 379-385. https://doi.org/10.1016/j.fsi.2007.08.008.

Gornati R, Papis E, Rimoldi S, Terova G, Saroglia M, Bernardini G, 2004. Rearing density influences the expression of stress-related genes in sea bass (Dicentrarchus labrax L.). Gene, 341: 111-118. https://doi.org/10.1016/j.gene.2004.06.020. 
Gupta RS, 1995. Phylogenetic analysis of the $90 \mathrm{kD}$ heat shock family of protein sequences and an examination of the relationship among animals, plants, and fungi species. Mol Biol Evol, 12: 1063-1073. https://doi.org/10.1093/oxfordjournals.molbev.a040281.

Iwama GK, Vijayan MM, Forsyth RB, Ackerman PA, 1999. Heat shock proteins and physiological stress in fish. Integr Comp Biol, 39: 901-909. https://doi.org/10.1093/icb/39.6.901.

Jiang B, Du JJ, Li YW, Ma P, Hu YZ, Li AX, 2019. Transcriptome analysis provides insights into molecular immune mechanisms of rabbitfish, Siganus oramin against Cryptocaryon irritans infection. Fish Shellfish Immunol, 88, 111-116. https://doi.org/10.1016/j.fsi.2019.02.039.

Jun Q, Hong Y, Hui W, Didlyn KM, Jie H, Pao X, 2015. Physiological responses and HSP70 mRNA expression in GIFT tilapia juveniles, Oreochromis niloticus under short-term crowding. Aquac Res, 46: 335-345. https://doi.org/10.1111/are.12189.

Kim JH, Dahms HU, Han KN, 2013. Biomonitoring of the river pufferfish, Takifugu obscurus in aquaculture at different rearing densities using stress-related genes. Aquac Res, 44: 1835-1846. https://doi.org/10.1111/j.1365-2109.2012.03189.x.

Liang YY, Guo HY, Zhu KC, Zhang N, Yang JW, Sun XX, Jiang SG, Zhang DC, 2018. Genomic structure and molecular characterization of growth hormone and its expression response to different feed types in golden pompano Trachinotus ovatus (linnaeus, 1758). Aquac Res, 49(5): 1973-1986. https://doi.org/10.1111/are.13653.

Lin X, Wu X, Liu X, 2018. Temperature stress response of heat shock protein 90 (Hsp90) in the clam Paphia undulata. Aquacult Fisheries, 3: 106-114. https://doi.org/10.1016/j.aaf.2018.04.003.

Livak KJ, Schmittgen TD, 2001. Analysis of relative gene expression data using realtime quantitative PCR and the $2^{-\triangle \Delta C T}$ method. Methods, 25: 402-408. https://doi.org/10.1006/meth.2001.1262.

Long L, Zhang H, Ni Q, Liu H, Wu F, Wang X, 2019. Effects of stocking density on growth, stress, and immune responses of juvenile Chinese sturgeon (Acipenser sinensis) in a recirculating aquaculture system. Comp Biochem Physiol C, 219: 25-34. https://doi.org/10.1016/j.cbpc.2019.02.002.

Manchado M, Salas-Leiton E, Infante C, Ponce M, Asensio E, Crespo A, Zuasti E, Cañavate JP, 2008. Molecular characterization, gene expression and transcriptional regulation of cytosolic HSP90 genes in the flatfish Senegalese sole (Solea senegalensis Kaup). Gene, 416: 77-84. https://doi.org/10.1016/j.gene.2008.03.007.

Ni M, Wen H, Li J, Chi M, Ren Y, Song Z, Ding H, 2014. Two HSPs gene from juvenile Amur sturgeon (Acipenser schrenckii): cloning, characterization and expression pattern to crowding and hypoxia stress. Fish Physiol Biochem, 40: 1801-1816. https://doi.org/10.1007/s10695-014-9969-9.

North BP, Turnbull JF, Ellis T, Porter MJ, Migaud H, Bron J, Bromage NR, 2006. The impact of stocking density on the welfare of rainbow trout (Oncorhynchus mykiss). Aquaculture, 255: 466-479. https://doi.org/10.1016/j.aquaculture.2006.01.004.

Patel HJ, Patel PD, Ochiana SO, Yan P, Sun W, Patel MR, Shah SK, Tramentozzi E, Brooks J, Bolaender A, Shrestha L, Stephani R, Finotti P, Leifer C, Li Z, Gewirth DT, Taldone T, Chiosis G, 2015. Structure-activity relationship in a purine-scaffold compound series with selectivity for the endoplasmic reticulum Hsp90 paralog, Grp94. J Med Chem, 58: 3922-3943. https://doi.org/10.1021/acs.jmedchem.5b00197.

Pierce A, Podlutskaya N, Halloran JJ, Hussong SA, Lin PY, Burbank R, Hart MJ, Galvan V, 2013. Over-expression of heat shock factor 1 phenocopies the effect of chronic inhibition of TOR by rapamycin and is sufficient to ameliorate Alzheimer's-like deficits in mice modeling the disease. J Neurochem, 124: 880-893. https://doi.org/10.1111/jnc. 12080.

Pratt WB, 1998. The hsp90-based chaperone system: involvement in signal transduction from a variety of hormone and growth factor receptors. Proc Soc Exp Biol Med, 217: 420434. https://doi.org/10.3181/00379727-217-44252.

Press CM, Evensen $\boldsymbol{\varnothing}, 1999$. The morphology of the immune system in teleost fishes. Fish Shellfish Immunol, 9(4): 309-318. https://doi.org/10.1006/fsim.1998.0181. 
Qin C, Shao Ting, Duan H, 2016. The cloning of a heat shock protein $90 \beta$ gene and expression analysis in Botia reevesae after ammonia- $\mathrm{N}$ exposure and Aeromonas hydrophila challenge. Aquacul Rep, 3: 159-165. https://doi.org/10.1016/j.aqrep.2016.02.004.

Rahimi MN, Buckton LK, Zaiter SS, Kho J, Chan V, Guo A, Konesan J, Kwon S, Lam LKO, Lawler MF, Leong M, Moldovan GD, Neale DA, Thornton G, McAlpine SR, 2018. Synthesis and structure-activity relationships of inhibitors that target the C-terminal MEEVD on heat shock protein 90. ACS Med Chem Lett, 9: 73-77. https://doi.org/10.1021/acsmedchemlett.7b00310.

Refaey MM, Li D, Tian X, Zhang Z, Zhang X, Li L, Tang R, 2018. High stocking density alters growth performance, blood biochemistry, intestinal histology, and muscle quality of channel catfish Ictalurus punctatus. Aquaculture, 492: 73-81. https://doi.org/10.1016/j.aquaculture.2018.04.003.

Roberts RJ, Agius C, Saliba C, Bossier P, Sung YY, 2010. Heat shock proteins (chaperones) in fish and shellfish and their potential role in relation to fish health: a review. J Fish Dis, 33:789-801. https://doi.org/10.1111/j.1365-2761.2010.01183.x.

Salas-Leiton E, Anguis V, Martín-Antonio B, Crespo D, Planas JV, Infante C, Cañavate JP, Manchado M, 2010. Effects of stocking density and feed ration on growth and gene expression in the Senegalese sole (Solea senegalensis): potential effects on the immune response. Fish Shellfish Immunol, 28: 296-302. https://doi.org/10.1016/j.fsi.2009.11.006.

Sanders BM, 1993. Stress proteins in aquatic organisms: an environmental perspective. Crit Rev Toxicol, 23(1): 49-75. https://doi.org/10.3109/10408449309104074.

Scheufler C, Brinker A, Bourenkov G, Pegoraro S, Moroder L, Bartunik H, HartI FU, Moarefi I, 2000. Structure of TPR domain-peptide complexes: critical elements in the assembly of the Hsp70-Hsp90 multichaperone machine. Cell, 101: 199-210. https://doi.org/10.1016/s0092-8674(00)80830-2.

Shin MK, Park HR, Yeo WJ, Han KN, 2018. Effects of thermal stress on the mRNA expression of SOD, HSP90, and HSP70 in the spotted sea bass (Lateolabrax maculatus). Ocean Sci J, 53: 1-10. https://doi.org/10.1007/s12601-018-0001-7.

Song HY, Dunbar JD, Zhang YX, Guo D, Donner DB, 1995. Identification of a protein with homology to hsp90 that binds the type 1 tumor necrosis factor receptor. J Biol Chem, 270: 3574-3581. https://doi.org/10.1074/jbc.270.8.3574.

Song C, Cui Y, Liu B, Xie J, Ge X, Xu P, Ren M, Miao L, Zhou Q, Lin Y, 2018. HSP60 and HSP90ß from blunt snout bream, Megalobrama amblycephala: molecular cloning, characterization, and comparative response to intermittent thermal stress and Aeromonas hydrophila infection. Fish Shellfish Immunol, 74: 119-132. https://doi.org/10.1016/j.fsi.2017.12.046.

Sreedhar AS, Kalmár E, Csermely P, Shen YF, 2004. Hsp90 isoforms: functions, expression and clinical importance. FEBS Lett, 562: 11-15. https://doi.org/10.1016/s0014-5793(04)00229-7.

Terova G, Gornati R, Rimoldi S, Bernardini G, Saroglia M, 2005. Quantification of a glucocorticoid receptor in sea bass (Dicentrarchus labrax, L.) reared at high stocking density. Gene, 357: 144-151. https://doi.org/10.1016/j.gene.2005.06.016.

Tutman P, Glavić N, Kožul V, Skaramuca B, Glamuzina B, 2004. Preliminary information on feeding and growth of pompano, Trachinotus Ovatus (Linnaeus, 1758) (Pisces; Carangidae) in captivity. Aquac Int, 12: 387-393. https://doi.org/10.1023/b:aqui.0000042135.88381.f4.

Valenzuela CA, Escobar-Aguirre S, Zuloaga R, Vera-Tobar T, Mercado L, Björnsson BT, Valdés JA, Molina A, 2019. Stocking density induces differential expression of immune-related genes in skeletal muscle and head kidney of fine flounder (Paralichthys adspersus). Vet Immunol Immunop, 210: https://doi.org/10.1016/j.vetimm.2019.03.004.

Vargas-Chacoff L, Muñoz JLP, Saravia J, Oyarzún R, Pontigo JP, González MP, Mardones O, Hawes C, Pino J, Wadsworth S, Morera FJ, 2019. Neuroendocrine stress 
response in Atlantic salmon (Salmo salar) and Coho salmon (Oncorynchus kisutch) during sea lice infestation. Aquaculture, 329-340. https://doi.org/10.1016/j.aquaculture.2019.04.046.

Vazzana M, Cammarata M, Cooper EL, Parrinello N, 2002. Confinement stress in sea bass (Dicentrarchus labrax) depresses peritoneal leukocyte cytotoxicity. Aquaculture, 210: 231-243. https://doi.org/10.1016/S0044-8486(01)00818-3.

Wang Q, Wang JN, Wang GL, Wu CD, Li JL, 2017. Molecular cloning, sequencing, and expression profiles of heat shock protein 90 (HSP90) in Hyriopsis cumingii exposed to different stressors: Temperature, cadmium and Aeromonas hydrophila. Aquaculture and Fisheries, 2: 59-66. https://doi.org/10.1016/j.aaf.2017.03.001.

Wei T, Gao Y, Wang R, Xu T, 2013. A heat shock protein $90 \beta$ isoform involved in immune response to bacteria challenge and heat shock from Miichthys miiuy. Fish Shellfish Immunol 35: 429-437. https://doi.org/10.1016/j.fsi.2013.04.045.

Wu CX, Zhao FY, Zhang Y, Zhu YJ, Ma MS, Mao HL, Hu CY, 2012. Overexpression of Hsp90 from grass carp (Ctenopharyngodon idella) increases thermal protection against heat stress. Fish Shellfish Immunol, 33: 42-47. https://doi.org/10.1016/j.fsi.2013.04.045. Yang Q, Zhu KC, Guo L, Liu BS, Guo HY, Zhang N, Yang JW, Zhang DC, 2020. Molecular characterization of GRP94 and HSP90a from Trachinotus ovatus, Linnaeus 1758 and their expression responses to various levels of stocking density stress and Cryptocaryon irritans infection. 529. https://doi.org/10.1016/j.aquaculture.2020.735601

Yebra-Pimentel ES, Gebert M, Jansen HJ, Jong-Raadsen SA, Dirks RPH, 2019. Deep transcriptome analysis of the heat shock response in an Atlantic sturgeon (Acipenser oxyrinchus) cell line. Fish Shellfish Immunol, 88: 508-517. https://doi.org/10.1016/j.fsi.2019.03.014.

Young JC, Moarefi I, Hartl FU, 2001. Hsp90: a specialized but essential protein-folding tool. J Cell Biol, 154: 267-273. https://doi.org/10.2307/1620585.

Zahedi S, Akbarzadeh A, Mehrzad J, Noori A, Harsij M, 2019. Effect of stocking density on growth performance, plasma biochemistry and muscle gene expression in rainbow trout (Oncorhynchus mykiss). Aquaculture, 271-278. https://doi.org/10.1016/j.aquaculture.2018.07.044.

Zhang XY, Zhang MZ, Zheng CJ, Liu J, Hu HJ, 2009. Identification of two hsp90 genes from the marine crab, Portunus trituberculatus and their specific expression profiles under different environmental conditions. Comp Biochem Physiol C, 150: 465-473. https://doi.org/10.1016/j.cbpc.2009.07.002.

Zhong ZH, Jiang B, Li ZC, Li SY, Li AX, 2020. Quantification of parasite abundance: A novel method to evaluate anti-Cryptocaryon irritans efficacy. Aquaculture, 528.

https://doi.org/10.1016/j.aquaculture.2020.735482

Zhou A, Xie S, Wang Z, Junaid M, Fan L, Wang C, Ye Q, Chen Y, Pei DS, Zou J, 2017. Molecular cloning, characterization and expression analysis of heat shock protein 90 in albino northern snakehead Channa argus. Gene, 626: 173-181. https://doi.org/10.1016/j.gene.2017.04.039.

Zhu KC, Liu BS, Zhang N, Guo HY, Guo L, Jiang SG, Zhang DC, 2020. Interferon regulatory factor 2 plays a positive role in interferon gamma expression in golden pompano, Trachinotus ovatus (Linnaeus 1758). Fish Shellfish Immunol, 96, 107-113. https://doi.org/10.1016/j.fsi.2019.12.006. 